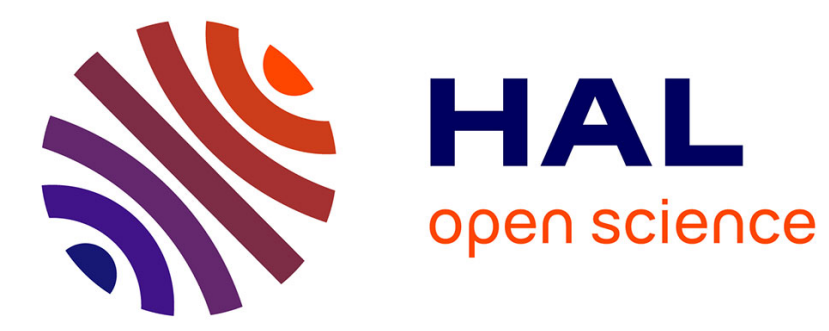

\title{
Optimal switching over multiple regimes
}

Huyen Pham, Vathana Ly Vath, Xunyu Zhou

\section{To cite this version:}

Huyen Pham, Vathana Ly Vath, Xunyu Zhou. Optimal switching over multiple regimes. 2007. hal00192151

\section{HAL Id: hal-00192151 \\ https://hal.science/hal-00192151}

Preprint submitted on 27 Nov 2007

HAL is a multi-disciplinary open access archive for the deposit and dissemination of scientific research documents, whether they are published or not. The documents may come from teaching and research institutions in France or abroad, or from public or private research centers.
L'archive ouverte pluridisciplinaire HAL, est destinée au dépôt et à la diffusion de documents scientifiques de niveau recherche, publiés ou non, émanant des établissements d'enseignement et de recherche français ou étrangers, des laboratoires publics ou privés. 


\title{
Optimal switching over multiple regimes
}

\author{
Huyên PHAM * Vathana Ly VATH ${ }^{\dagger}$ Xun Yu ZHOU ${ }^{\ddagger}$
}

November 27, 2007

\begin{abstract}
This paper studies the optimal switching problem for a general one-dimensional diffusion with multiple (more than two) regimes. This is motivated in the real options literature by the investment problem of a firm managing several production modes while facing uncertainties. A viscosity solutions approach is employed to carry out a fine analysis on the associated system of variational inequalities, leading to sharp qualitative characterizations of the switching regions. These characterizations, in turn, reduce the switching problem into one of finding a finite number of threshold values in state that would trigger switchings. The results of our analysis take several qualitatively different forms depending on model parameters, and the issue of when and where it is optimal to switch is addressed. The general results are then demonstrated by the threeregime case, where a quasi-explicit solution is obtained, and a numerical procedure to find these critical values is devised in terms of the expectation functionals of hitting times for one-dimensional diffusions.
\end{abstract}

Key words : Optimal multiple switching, variational inequalities, switching regions, viscosity solutions, hitting times of Itô diffusion, real options.

MSC 2000 subject classification : 60G40, 93E20, 49L25.

\section{Introduction}

Optimal multiple switching is the problem of determining an optimal sequence of stopping times for a stochastic process with several regimes (or modes). This is a classical and important problem, extensively studied since the late seventies. It has recently received renewed and increasing interest due to many applications in economics and finance, especially to real options. Actually, optimal switching provides a suitable model to capture the

*Laboratoire de probabilités et modèles aléatoires, Universités Paris 6-Paris 7, 2 Place Jussieu, 75251 Paris cedex 05, France, CREST and Institut Universitaire de France, pham@math.jussieu.fr.

${ }^{\dagger}$ ENSIIE, 18 allée Jean Rostand, 91025 Evry Cedex, France, and Université d'Evry Val d'Essonne, lyvath@ensiie.fr.

${ }^{\ddagger}$ Mathematical Institute and Nomura Centre for Mathematical Finance, University of Oxford, 24-29 St Giles', Oxford OX1 3LB, UK, and Department of Systems Engineering and Engineering Management, The Chinese University of Hong Kong, Shatin, Hong Kong. zhouxy@maths.ox.ac.uk. Supported in part by a start-up fund at University of Oxford, and RGC Earmarked Grants CUHK418605 and CUHK418606. 
value of managerial flexibility in making decisions under uncertainty, and has been used in the pioneering works by Brennan and Schwarz [5] for resource extraction, and Dixit [9] for production facility problems.

The optimal two-regime switching problem has been the most largely studied in the literature, and is often referred to as the starting-and-stopping problem : in the case of a geometric Brownian motion and some special profit functions on infinite horizon, Brekke and Oksendal [4], Duckworth and Zervos [11], and Zervos [19] apply a verification approach for solving the variational inequality associated with this impulse control problem (see Bensoussan and Lions [2] or Tang and Yong [18]). Various extensions of this model are solved in Pham and Vath [17]. Hamadène and Jeanblanc [14] consider a finite horizon starting-andstopping problem by using reflected backward stochastic differential equations (BSDEs). Bayraktar and Egami [1] employ optimal stopping theory for studying the optimal tworegime switching problem on infinite horizon for one-dimensional diffusions. In this latter framework, Guo and Tomecek [13] solve some special cases by connecting optimal switching to singular control problem.

The applications of the starting-and-stopping problem to real options, for example the management of a power plant, are limited to the case of two modes, e.g. operating and closed. In practice, however, the efficient management of a power plant requires more than two production modes to include intermediate operating modes corresponding to different subsets of turbine running. Such an example of multiple switching problems applied to energy tolling agreements was considered by Carmona and Ludkowski [6], and Deng and Xia [8], who focus mainly on a numerical resolution based on Monte-Carlo regressions. Yet, there is little work addressing a complete treatment and mathematical resolution of the optimal multiple switching problem. The difficulty with such a problem is evident: In sharp contrast with the two-regime problem, a multiple switching problem needs to decide not only when to switch, but also where to switch. Recently, Djehiche, Hamadène and Popier [10], and Hu and Tang [15] have studied optimal multiple switching problems for general adapted processes by means of reflected BSDEs, and they are mainly concerned with the existence and uniqueness of solution to these reflected BSDEs. However, the important issue as to which regime to optimally switch has been left completely open.

In this paper, we consider the optimal multiple switching problem on infinite horizon for a general one-dimensional diffusion. The multiple regimes are differentiated via their profit functions, which are of very general form. The transition from one regime to another one is realized sequentially at random times (which are part of the decisions), and incurs a fixed cost. Our objective is to provide an explicit characterization of the switching regions showing when and where it is optimal to change the regime. We adopt a direct solution method via the viscosity solutions technique. By carrying out an a priori and fine analysis of the system of variational inequalities associated with the optimal switching problem, we give a sharp qualitative description of the switching regions. Specifically, we give conditions under which one should switch to a regime with higher profit, and to a regime with lower profit, and we identify these destination regimes. The switching regions take various structures, depending on model parameters via explicit conditions, which have meaningful economic interpretations. We showcase our general results by the 
three-regime case, where we present a complete picture of the situations as to when and where it is optimal to switch, and we reduce the problem into one of finding a finite number of threshold values of the switching regions. We also design an algorithm to compute these critical values based on the computations of expectation functionals of hitting times for one-dimensional diffusions.

The rest of the paper is organized as follows. In Section 2, we formulate precisely the optimal multiple switching problem. We recall in Section 3 the system of variational inequalities and the boundary data that characterize theoretically the value functions in the viscosity sense. The continuous differentiability of the value functions serves also as an important result in our subsequent analysis. Section 4 is devoted to the qualitative description of the switching regions. In the three-regime case considered in Section 5, we give a complete solution by reducing the original switching problem into one of finding a finite number of threshold values of the switching regions. Finally, in Section 6, we provide a numerical procedure for computing these critical values.

\section{Model and problem formulation}

\subsection{General setup and assumptions}

We present our general model and emphasize the key assumptions. The state process $X$ is a one-dimensional diffusion on $(0, \infty)$ whose dynamic is given by :

$$
d X_{t}=b\left(X_{t}\right) d t+\sigma\left(X_{t}\right) d W_{t}
$$

where $W$ is a standard Brownian motion on a filtered probability space $\left(\Omega, \mathcal{F}, \mathbb{F}=\left(\mathcal{F}_{t}\right)_{t \geq 0}, P\right)$ satisfying the usual conditions, and $b, \sigma$ are measurable functions on $(0, \infty)$. We assume that the $\operatorname{SDE}(2.1)$ has a unique strong solution, denoted by $X^{x}$, given an initial condition $X_{0}=x \in(0, \infty)$. The coefficients $b$ and $\sigma$ satisfy the growth condition

$$
x b(x), \frac{1}{2} \sigma^{2}(x) \leq C\left(1+x^{2}\right), \quad \forall x>0,
$$

for some positive constant $C$, and the nondegeneracy condition :

$$
\sigma(x)>0, \quad \forall x>0
$$

We also make the standing assumption that 0 and $\infty$ are natural boundaries for $X$ (see e.g. [3] for boundary classification of one-dimensional diffusions), and for all $t \geq 0$,

$$
\left.\left.X_{t}^{x} \longrightarrow 0 \text { (resp. } \infty\right) \text {, a.s. } \quad \text { as } x \text { goes to } 0 \text { (resp. } \infty\right) .
$$

Throughout the paper, we denote by $\mathcal{L}$ the infinitesimal generator of the diffusion $X$, i.e.

$$
\mathcal{L} \varphi(x)=b(x) \varphi^{\prime}(x)+\frac{1}{2} \sigma^{2}(x) \varphi^{\prime \prime}(x) .
$$

For any positive constant $r$, the ordinary differential equation of second order :

$$
r \varphi-\mathcal{L} \varphi=0
$$


has two linearly independent solutions. These solutions are uniquely determined (up to a multiplication), if we require one of them to be increasing and the other decreasing. We denote by $\psi_{r}^{+}$the increasing solution and $\psi_{r}^{-}$the decreasing solution. They are called fundamental solutions of (2.5) and all other solutions can be expressed as their linear combinations. Moreover, since 0 and $\infty$ are natural boundaries for $X$, we have (see [3]) :

$$
\psi_{r}^{+}(0)=\psi_{r}^{-}(\infty)=0, \quad \psi_{r}^{+}(\infty)=\psi_{r}^{-}(0)=\infty .
$$

In the sequel, $r$ will be fixed, and hence we omit the dependence in $r$ by setting $\psi^{+}=\psi_{r}^{+}$ and $\psi^{-}=\psi_{r}^{-}$.

\section{Canonical examples}

Our two basic examples in finance for $X$ satisfying the above assumptions are

- a geometric Brownian motion (GBM)

$$
d X_{t}=\mu X_{t} d t+\vartheta X_{t} d W_{t}
$$

where $\mu$ and $\vartheta$ are two constants with $\vartheta>0$. Condition (2.4) is clearly satisfied. Moreover, the two fundamental solutions of (2.5) are given by $\psi^{-}(x)=x^{m^{-}}, \psi^{+}(x)$ $=x^{m^{+}}$, where $m^{-}<0<m_{+}$are the roots of $\frac{1}{2} \vartheta^{2} m^{2}+\left(\mu-\frac{1}{2} \vartheta^{2}\right) m-r=0$.

- a geometric mean-reverting (GMR) process

$$
d X_{t}=\mu X_{t}\left(\bar{y}-\ln X_{t}\right) d t+\vartheta X_{t} d W_{t}
$$

where $\mu, \bar{y}, \vartheta$ are constants with $\mu, \vartheta>0$. This last example for $X$ is well suited for modelling commodity and electricity prices since they are expected to be long-term stationary. By the change of variable $Y_{t}=e^{\mu t} \ln X_{t}$, we easily see by Itô's formula that $Y_{t}$ follows a geometric brownian motion, and so condition (2.4) is satisfied.

The operational regimes are characterized by their running reward functions $f_{i}: \mathbb{R}_{+}$ $\rightarrow \mathbb{R}, i \in \mathbb{I}_{d}=\{1, \ldots, d\}$. We assume that for each $i \in \mathbb{I}_{d}$, the function $f_{i}$ is nonnegative, without loss of generality (w.l.o.g.) $f_{i}(0)=0$, and satisfies the linear growth condition:

$$
f_{i}(x) \leq C(1+|x|), \quad \forall x \in \mathbb{R}_{+},
$$

for some positive constant $C$. The numbering $i=1, \ldots, d$, on the regimes is ordered by increasing level of profitability, which roughly means that the sequence of functions $f_{i}$ is increasing in $i$. The ordering condition on the profit functions will be detailed later.

Switching from regime $i$ to $j$ incurs an instantaneous cost, denoted by $g_{i j}$, with the convention $g_{i i}=0$. The following triangular condition is reasonable:

$$
g_{i k}<g_{i j}+g_{j k}, \quad j \neq i, k
$$

which means that it is less expensive to switch directly in one step from regime $i$ to $k$ than in two steps via an intermediate regime $j$. Notice that a switching cost $g_{i j}$ may be negative, and condition (2.9) for $i=k$ prevents an arbitrage by simply switching back and forth, i.e.

$$
g_{i j}+g_{j i}>0, \quad i \neq j \in \mathbb{I}_{d} .
$$




\subsection{The optimal switching problem}

A decision (strategy) for the operator is an impulse control $\alpha$ consisting of a double sequence $\tau_{1}, \ldots, \tau_{n}, \ldots, \iota_{1}, \ldots, \iota_{n}, \ldots, n \in \mathbb{N}^{*}=\mathbb{N} \backslash\{0\}$, where $\tau_{n}, \mathbb{N} \backslash\{0\}$, are $\mathbb{F}$-stopping times in $[0, \infty]$, denoted by $\tau_{n} \in \mathcal{T}, \tau_{n}<\tau_{n+1}$ and $\tau_{n} \rightarrow \infty$ a.s., representing the decision on "when to switch", and $\iota_{n}$ are $\mathcal{F}_{\tau_{n}}$-measurable valued in $\mathbb{I}_{d}$, representing the new value of the regime at time $\tau_{n}$ until time $\tau_{n+1}$ or the decision on "where to switch". We denote by $\mathcal{A}$ the set of all such impulse controls. Given an initial regime value $i \in \mathbb{I}_{d}$, and a control $\alpha=\left(\tau_{n}, \iota_{n}\right)_{n \geq 1}$ $\in \mathcal{A}$, we denote

$$
I_{t}^{i}=\sum_{n \geq 0} \iota_{n} 1_{\left[\tau_{n}, \tau_{n+1}\right)}(t), \quad t \geq 0, \quad I_{0^{-}}^{i}=i,
$$

which is the piecewise constant process indicating the regime value at any time $t$. Here, we set $\tau_{0}=0$ and $\iota_{0}=i$. We notice that $I^{i}$ is a cadlag process, possibly with a jump at time 0 if $\tau_{1}=0$ and so $I_{0}^{i}=\iota_{1}$.

The expected total profit of running the system when initial state is $(x, i)$ and using the impulse control $\alpha=\left(\tau_{n}, \iota_{n}\right)_{n \geq 1} \in \mathcal{A}$ is

$$
J_{i}(x, \alpha)=E\left[\int_{0}^{\infty} e^{-r t} f_{I_{t}^{i}}\left(X_{t}^{x}\right) d t-\sum_{n=1}^{\infty} e^{-r \tau_{n}} g_{\iota_{n-1}, \iota_{n}}\right] .
$$

Here $r>0$ is a positive discount factor, and we use the convention that $e^{-r \tau_{n}(\omega)}=0$ when $\tau_{n}(\omega)=\infty$. The objective is to maximize this expected total profit over $\mathcal{A}$. Accordingly, we define the value functions

$$
v_{i}(x)=\sup _{\alpha \in \mathcal{A}} J_{i}(x, \alpha), \quad x>0, i \in \mathbb{I}_{d}
$$

We shall see later that for $r$ large enough, the expectation defining $J_{i}(x, \alpha)$ is well-defined and the value function $v_{i}$ is finite.

\section{Dynamic programming PDE characterization}

In this section, we state some general PDE characterization of the value functions by using the dynamic programming approach.

We first state the linear growth property and the boundary condition on the value functions.

Lemma 3.1 There exists some positive constant $\rho$ such that for $r>\rho$, the value functions $v_{i}, i \in \mathbb{I}_{d}$, are finite on $(0, \infty)$. In this case, the value functions $v_{i}, i \in \mathbb{I}_{d}$, satisfy a linear growth condition

$$
0 \leq v_{i}(x) \leq K(1+x), \quad \forall x>0
$$

for some positive constant $K$. Moreover, we have for all $i \in \mathbb{I}_{d}$,

$$
v_{i}\left(0^{+}\right):=\lim _{x \downarrow 0} v_{i}(x)=\max _{j \in \mathbb{I}_{d}}\left(-g_{i j}\right) .
$$


Proof. We first show the finiteness of the value functions and their linear growth. By induction, we obtain that for all $N \geq 1, \tau_{1} \leq \ldots \leq \tau_{N}, \kappa_{0}=i, \kappa_{n} \in \mathbb{I}_{d}, n=1, \ldots, N$ (see [17]) :

$$
-\sum_{n=1}^{N} e^{-r \tau_{n}} g_{\kappa_{n-1}, \kappa_{n}} \leq \max _{j \in \mathbb{I}_{d}}\left(-g_{i j}\right), \quad \text { a.s. }
$$

By definition, using the latter inequality and the growth condition (2.8), we have for all $i$ $\in \mathbb{I}_{d}, x>0$, and $\alpha \in \mathcal{A}$ :

$$
\begin{aligned}
J_{i}(x, \alpha) & \leq E\left[\int_{0}^{\infty} e^{-r t} f_{I_{t}^{i}}\left(X_{t}^{x}\right) d t+\max _{j \in \mathbb{I}_{d}}\left(-g_{i j}\right)\right] \\
& \leq E\left[\int_{0}^{\infty} e^{-r t} C\left(1+\left|X_{t}^{x}\right|\right) d t+\max _{j \in \mathbb{I}_{d}}\left(-g_{i j}\right)\right] .
\end{aligned}
$$

Now, a standard estimate on the process $\left(X_{t}\right)_{t \geq 0}$, based on Itô's formula and Gromwall's lemma, yields

$$
E\left[\left|X_{t}^{x}\right|\right] \leq E\left[\left(X_{t}^{x}\right)^{2}\right]^{\frac{1}{2}} \leq e^{2 C t}(1+x)
$$

for some positive constant $C$ (independent of $t$ and $x$ ). Plugging the above inequality into (3.1), and from the arbitrariness of $\alpha \in \mathcal{A}$, we get

$$
v_{i}(x) \leq \frac{C}{r}+C(1+x) \int_{0}^{\infty} e^{(2 C-r) t} d t+\max _{j \in \mathbb{I}_{d}}\left(-g_{i j}\right) .
$$

We therefore have the finiteness of the value functions if $r>2 C$, in which case the value functions satisfy the linear growth condition.

We now turn to the boundary data at 0 of the value functions. By considering the particular strategy $\tilde{\alpha}=\left(\tilde{\tau}_{n}, \tilde{\kappa}_{n}\right)$ of immediately switching from the initial state $(x, i)$ to state $(x, j), j \in \mathbb{I}_{d}$, at cost $g_{i j}$ and then doing nothing, i.e. $\tilde{\tau}_{1}=0, \tilde{\kappa}_{1}=j, \tilde{\tau}_{n}=\infty, \tilde{\kappa}_{n}=$ $j$ for all $n \geq 2$, we have

$$
J_{i}(x, \tilde{\alpha})=E\left[\int_{0}^{\infty} e^{-r t} f_{j}\left(\tilde{X}_{t}^{x, j}\right) d t-g_{i j}\right]
$$

where $\tilde{X}^{x, j}$ denotes the controlled process in regime $j$ starting from $x$ at time 0 . Since $f_{j}$ is nonnegative, and by the arbitrariness of $j$, we obtain:

$$
0 \leq \max _{j \in \mathbb{I}_{d}}\left(-g_{i j}\right) \leq v_{i}(x)
$$

To obtain the reverse inequality, we introduce the conjugate of $f_{i}$ :

$$
\tilde{f}_{i}(y):=\sup _{x \geq 0}\left[f_{i}(x)-x y\right]<\infty, \quad \forall y>0,
$$

and follow the proof of Lemma 3.1 in [17] to obtain for all $x, y>0$, and $\alpha \in \mathcal{A}$,

$$
\begin{aligned}
J_{i}(x, \alpha) & \leq E\left[\int_{0}^{\infty} e^{-r t}\left(y X_{t}^{x}+\tilde{f}(y)\right) d t+\max _{j \in \mathbb{I}_{d}}\left(-g_{i j}\right)\right] \\
& =\int_{0}^{\infty} e^{-r t} y E\left[X_{t}^{x}\right] d t+\int_{0}^{\infty} e^{-r t} \tilde{f}(y) d t+\max _{j \in \mathbb{I}_{d}}\left(-g_{i j}\right) .
\end{aligned}
$$


By sending $x$ to zero and then $y$ to infinity, and using (2.4) and recalling that $\tilde{f}_{i}(\infty)=$ $f_{i}(0)=0$ for $i \in \mathbb{I}_{d}$, we have $J_{i}\left(0^{+}, \alpha\right) \leq \max _{j \in \mathbb{I}_{d}}\left(-g_{i j}\right)$. From the arbitrariness of $\alpha$, we obtain

$$
v_{i}\left(0^{+}\right) \leq \max _{j \in \mathbb{I}_{d}}\left(-g_{i j}\right)
$$

which concludes our proof.

Remark 3.1 The original problem is called well-posed if the value functions are finite (otherwise, ill-posed). An ill-posed problem is a wrongly modelled problem where the trade-off is not set right so it is possible to push the profit to arbitarily high. The preceding result indicates that the discount rate must be sufficiently high to avoid a wrong problem.

However, for the GMR case (see (2.7)), the problem is well-posed so long as $r>0$, which is due to the mean-reverting properties of the GMR state process itself. Indeed, by Itô's formula, we have :

$$
E\left[\left|X_{t}^{x}\right|^{2}\right]=x^{2}+\int_{0}^{t}\left(2 \mu \bar{y}+\nu-2 \mu \ln X_{t}^{x}\right)\left|X_{t}^{x}\right|^{2} d t
$$

A basic study of the function $x \rightarrow(2 \mu \bar{y}+\nu-2 \mu \ln x) x^{2}$ shows that it admits a global maximum point. As such, there exists some constant $K$ such that

$$
E\left[\left|X_{t}^{x}\right|^{2}\right] \leq x^{2}+K t
$$

Using the same arguments as in the proof of Lemma 3.1, we thus obtain the finiteness of the value functions when $r>0$.

The dynamic programming principle combined with the notion of viscosity solutions are known to be a general and powerful tool for characterizing the value function of a stochastic control problem via a PDE representation. In our context, we have the following $\mathrm{PDE}$ characterization on the value functions.

Theorem 3.1 The value functions $v_{i}, i \in \mathbb{I}_{d}$, are the unique viscosity solutions to the system of variational inequalities :

$$
\min \left\{r v_{i}-\mathcal{L}_{i} v_{i}-f_{i}, v_{i}-\max _{j \neq i}\left(v_{j}-g_{i j}\right)\right\}=0, \quad x \in(0, \infty), \quad i \in \mathbb{I}_{d},
$$

in the following sense:

(1) Viscosity property. For each $i \in \mathbb{I}_{d}, v_{i}$ is a viscosity solution to

$$
\min \left\{r v_{i}-\mathcal{L}_{i} v_{i}-f_{i}, v_{i}-\max _{j \neq i}\left(v_{j}-g_{i j}\right)\right\}=0, \quad x \in(0, \infty) .
$$

(2) Uniqueness property. If $w_{i}, i \in \mathbb{I}_{d}$, are viscosity solutions with linear growth conditions on $(0, \infty)$ and boundary conditions $w_{i}\left(0^{+}\right)=\max _{j \in \mathbb{I}_{d}}\left[-g_{i j}\right]$ to the system of variational inequalities $(3.2)$, then $v_{i}=w_{i}$ on $(0, \infty)$. 
Remark 3.2 (1) For fixed $i \in \mathbb{I}_{d}$, we also have uniqueness of viscosity solution to equation (3.3) in the class of continuous functions with linear growth condition on $(0, \infty)$ and given boundary condition on 0 . In the next sections, we shall use either uniqueness of viscosity solutions to the system (3.2) or for fixed $i$ to equation (3.3).

(2) The viscosity property is proved in [16]. The uniqueness result is proved in [17] when $X$ is a geometric brownian motion. A straightforward modification of their proof, see their Step 1 in the construction of a strict supersolution, provides the result for general one-dimensional diffusion satisfying condition (2.2).

(3) For fixed $i \in \mathbb{I}_{d}$, and by setting $h_{i}=\max _{j}\left(v_{j}-g_{i j}\right)$, we notice from the free-boundary characterization (3.2) that $v_{i}$ may be represented as the value function of the optimal stopping problem :

$$
v_{i}(x)=\sup _{\tau \in \mathcal{T}} E\left[\int_{0}^{\tau} e^{-r t} f_{i}\left(X_{t}^{x}\right) d t+e^{-r \tau} h_{i}\left(X_{\tau}^{x}\right)\right], \quad x>0 .
$$

We also quote the useful smooth fit property on the value functions, proved in [16].

Theorem 3.2 For all $i \in \mathbb{I}_{d}$, the value function $v_{i}$ is continuously differentiable on $(0, \infty)$.

For any regime $i \in \mathbb{I}_{d}$, we introduce the switching region :

$$
\mathcal{S}_{i}=\left\{x \in(0, \infty): v_{i}(x)=\max _{j \neq i}\left(v_{j}-g_{i j}\right)(x)\right\} .
$$

$\mathcal{S}_{i}$ is a closed subset of $(0, \infty)$ and corresponds to the region where it is optimal for the controller to change regime. The complement set $\mathcal{C}_{i}$ of $\mathcal{S}_{i}$ in $(0, \infty)$ is the so-called continuation region :

$$
\mathcal{C}_{i}=\left\{x \in(0, \infty): v_{i}(x)>\max _{j \neq i}\left(v_{j}-g_{i j}\right)(x)\right\},
$$

where it is optimal to stay in regime $i$. In this open domain, the value function $v_{i}$ is smooth $\left(C^{2}\right.$ on $\left.\mathcal{C}_{i}\right)$ and satisfies in a classical sense :

$$
r v_{i}(x)-\mathcal{L}_{i} v_{i}(x)-f_{i}(x)=0, \quad x \in \mathcal{C}_{i} .
$$

Remark 3.3 There are no isolated points in a switching region $\mathcal{S}_{i}$ : for any $x_{0} \in \mathcal{S}_{i}$, there exists some $\varepsilon>0$ s.t. either $\left(x_{0}-\varepsilon, x_{0}\right)$ or $\left(x_{0}, x_{0}+\varepsilon\right)$ is included in $\mathcal{S}_{i}$. Indeed, otherwise, recalling that $\mathcal{C}_{i}=(0, \infty) \backslash \mathcal{S}_{i}$ is open, one could find some $\varepsilon>0$ s.t. $\left(x_{0}-\varepsilon, x_{0}\right) \cup\left(x_{0}, x_{0}+\varepsilon\right)$ $\subset \mathcal{C}_{i}$. Hence, on $\left(x_{0}-\varepsilon, x_{0}\right) \cup\left(x_{0}, x_{0}+\varepsilon\right), v_{i}$ satisfies : $r v_{i}-\mathcal{L} v_{i}-f_{i}=0$. By the smooth-fit property of $v_{i}$ at $x_{0}$, this implies that $v_{i}$ is actually $C^{2}$ at $x_{0}$ and satisfies $r v_{i}-\mathcal{L} v_{i}-f_{i}=$ 0 on $\left(x_{0}-\varepsilon, x_{0}+\varepsilon\right)$. Hence, $x_{0}$ lies in $\mathcal{C}_{i}$, a contradiction.

Following the general theory of optimal stopping and dynamic programming principle, see e.g. [12], we introduce the sequence of stopping times and regime decisions for $v_{i}(x)$ :

$$
\begin{aligned}
\tau_{1}^{*}=\inf \left\{t \geq 0: X_{t}^{x} \in \mathcal{S}_{i}\right\}, & \iota_{1}^{*} \in \arg \max _{j \neq i}\left(v_{j}-g_{i j}\right)\left(X_{\tau_{1}^{*}}^{x}\right) \\
& \vdots \\
\tau_{n}^{*}=\inf \left\{t \geq \tau_{n-1}^{*}: X_{t}^{x} \in \mathcal{S}_{\iota_{n-1}^{*}}\right\}, & \iota_{n}^{*} \in \arg \max _{j \neq \iota_{n-1}^{*}}\left(v_{j}-g_{\iota_{n-1}^{*}, j}\right)\left(X_{\tau_{n-1}^{*}}^{x}\right) .
\end{aligned}
$$


The condition

$$
\limsup _{x \rightarrow \infty} \frac{x}{\psi^{+}(x)}=0
$$

ensures that the sequence given in (3.6)-(3.7) is optimal for $v_{i}(x)$. Indeed, from the linear growth condition on the value functions, and with the notation of Remark $3.2(\mathbf{3})$, we have $\lim \sup _{x \rightarrow \infty} \frac{\max \left(h_{i}(x), 0\right)}{\psi^{+}(x)}=0$, and this implies from [7] that $\tau_{1}^{*}$ is an optimal stopping time for (3.4). By induction, we obtain that $\left(\tau_{n}^{*}, \iota_{n}^{*}\right)_{n \geq 1}$ is an optimal sequence for $v_{i}(x)$. In the remainder of the paper, we assume that (3.8) is satisfied.

Remark 3.4 Notice that condition (3.8) is satisfied for $r>\mu$ in the example of a GBM, since in this case $\psi^{+}(x)=x^{m^{+}}$with $m^{+}>1$. We now check for the case of a GMR process. In this case as defined in (2.7), the ODE (2.5) becomes

$$
r \varphi-\mu x(\bar{y}-\ln x) \varphi^{\prime}-\frac{1}{2} \nu^{2} x^{2} \varphi^{\prime \prime}=0 .
$$

By a change of variable $z=\ln x+\frac{\beta}{\delta}$, the preceding equation becomes

$$
\varphi^{\prime \prime}-\delta z \varphi^{\prime}-\gamma \varphi=0
$$

where $\beta=1-\frac{2 \mu \bar{y}}{\nu^{2}}, \delta=\frac{2 \mu}{\nu^{2}}>0$, and $\gamma=\frac{2 r}{\nu^{2}}>0$. Using power series as likely candidate solutions to our ODE., we obtain two linearly independent solutions $\phi_{1}$ and $\phi_{2}$ described as follows :

$$
\phi_{1}(z)=\sum_{n=0}^{\infty} a_{2 n} z^{2 n} \text { and } \phi_{2}(z)=\sum_{n=0}^{\infty} a_{2 n+1} z^{2 n+1},
$$

where $a_{0}=a_{1}=1, a_{2 n+2}=\frac{\Pi_{k=0}^{n}(2 k \alpha+\gamma)}{(2 n+2) !}>0$, and $a_{2 n+3}=\frac{\Pi_{k=0}^{n}[(2 k+1) \alpha+\gamma]}{(2 n+3) !}>0$, for all $n \geq 2$. The radius of convergence of both functions $\phi_{1}$ and $\phi_{2}$ are infinite, as such they are of class $C^{\infty}$. Let us now denote by $\chi^{+}$, one of the two fundamental solutions to (3.10), which is non-decreasing and satisfies

$$
\lim _{z \rightarrow+\infty} \chi^{+}(z)=+\infty, \quad \lim _{z \rightarrow-\infty} \chi^{+}(z)=0 .
$$

$\chi^{+}$could therefore be written as a linear combination of $\phi_{1}$ and $\phi_{2}$ :

$$
\chi^{+}=A \phi_{1}+B \phi_{2}
$$

where we see that $A$ and $B$ should be strictly non-positive due to condition (3.12) and the fact that $\phi_{1}$ and $\phi_{2}$ are respectively even and odd functions. Notice that proving that $\psi^{+}$, one of the two fundamental solutions of (3.9) as described by (2.6), satisfies condition (3.8) is equivalent to proving that

$$
\lim _{z \rightarrow+\infty} \frac{e^{z}}{\chi^{+}(z)}=0
$$

Since $\chi^{+}(z) \geq \min (A, B)\left(\phi_{1}+\phi_{2}\right)(z)$, it suffices to show there exists some $\varepsilon>0$ such that for all $z$ large enough,

$$
\phi_{1}(z)+\phi_{2}(z) \geq e^{(1+\varepsilon) z} .
$$


Actually, fix some $\varepsilon>0$, and develop the function $e^{(1+\varepsilon) z}$ into power series. Then, by using the Stirling formula, we may compare the coefficients $b_{n}=\frac{(1+\varepsilon)^{n}}{n !}$ of the latter power series with the coefficients $a_{n}$ of $\left(\phi_{1}+\phi_{2}\right)$. We obtain $b_{n}<a_{n}$ for $n$ sufficiently large enough, which shows (3.14), and hence (3.13) or (2.6).

We end this section with some notation. We introduce the functions

$$
\hat{V}_{i}(x)=E\left[\int_{0}^{\infty} e^{-r t} f_{i}\left(X_{t}^{x}\right) d t\right], \quad x>0, i \in \mathbb{I}_{d},
$$

which are particular solutions to

$$
r w-\mathcal{L} w-f_{i}=0 .
$$

All other solutions to (3.16) are in the form

$$
w=\hat{V}_{i}+A \psi^{-}+B \psi^{+}
$$

for some constants $A$ and $B$.

Remark 3.5 Notice that $\hat{V}_{i}$ corresponds to the expected profit $J_{i}(x, \alpha)$ where $\alpha$ is the strategy of never switching. In particular, we obviously have $v_{i} \geq \hat{V}_{i}$. Moreover, from definition (3.15) and condition (2.4) together with (Hf) to be introduced below, we may apply monotone convergence theorem to get :

$$
\lim _{x \rightarrow \infty}\left(\hat{V}_{j}-\hat{V}_{i}\right)(x)=\frac{\left(f_{j}-f_{i}\right)(\infty)}{r}, \quad \forall i, j \in \mathbb{I}_{d}
$$

\section{Qualitative properties of the switching regions}

In this section, we focus on the qualitative aspects on deriving the solution to the switching problem. Basically, we raise the following questions : When and where does one switch? In view of the general dynamic programming results stated in the previous section, the answer to these questions is provided by the description of the switching regions $S_{i}$ and the determination of the argument maximum in $v_{i}(x)=\max _{j \neq i}\left(v_{j}-g_{i j}\right)(x), i \in \mathbb{I}_{d}$.

From the definition (3.5) of the switching regions, we have the elementary decomposition property :

$$
\mathcal{S}_{i}=\cup_{j \neq i} \mathcal{S}_{i j}, \quad i \in \mathbb{I}_{d},
$$

where

$$
\mathcal{S}_{i j}=\left\{x \in(0, \infty): v_{i}(x)=\left(v_{j}-g_{i j}\right)(x)\right\}
$$

is the switching region from regime $i$ to regime $j$. Moreover, from condition (2.9), when one switch from regime $i$ to regime $j$, one does not switch immediately to another regime, i.e. one stays for a while in the continuation region of regime $j$. In other words,

$$
\mathcal{S}_{i j} \subset \mathcal{C}_{j}, \quad j \neq i \in \mathbb{I}_{d} .
$$

The following useful lemma gives some partial information about the structure of the switching regions. 
Lemma 4.1 For all $i \neq j$ in $\mathbb{I}_{d}$, we have

$$
\mathcal{S}_{i j} \subset Q_{i j}:=\left\{x>0:\left(f_{j}-f_{i}\right)(x)-r g_{i j} \geq 0\right\} .
$$

Proof. Let $x \in \mathcal{S}_{i j}$. By setting $\varphi_{j}=v_{j}-g_{i j}$, it follows that $x$ is a minimum of $v_{i}-\varphi_{j}$ with $v_{i}(x)=\varphi_{j}(x)$. Moreover, since $x$ lies in the open set $\mathcal{C}_{j}$ where $v_{j}$ is smooth, we have that $\varphi_{j}$ is $C^{2}$ in a neighborhood of $x$. By the supersolution viscosity property of $v_{i}$ to the PDE (3.2), this yields :

$$
r \varphi_{j}(x)-\mathcal{L} \varphi_{j}(x)-f_{i}(x) \geq 0
$$

Now recall that for $x \in \mathcal{C}_{j}$, we have

$$
r v_{j}(x)-\mathcal{L} v_{j}(x)-f_{j}(x)=0
$$

so by substituting into (4.1), we obtain :

$$
\left(f_{j}-f_{i}\right)(x)-r g_{i j} \geq 0
$$

which is the required result.

\section{Economic assumptions on profit functions and switching costs}

We now formalize the differentiation between the operational regimes. We consider the following ordering conditions on the regimes through their reward functions :

$$
f_{1} \prec f_{2} \prec \ldots \prec f_{d}
$$

$\Longleftrightarrow$

for all $i<j \in \mathbb{I}_{d}, f_{j}-f_{i}$ is decreasing on $\left(0, \hat{x}_{i j}\right)$ and increasing on $\left(\hat{x}_{i j}, \infty\right)$ for some $\hat{x}_{i j} \in \mathbb{R}_{+}$.

Economically speaking, the ordering condition $f_{i} \prec f_{j}$ means that the profit in regime $j>i$ is "better" than profit in regime $i$ from a certain level, and the improvement becomes then better and better, possibly with a saturation when $\left(f_{j}-f_{i}\right)(\infty)<\infty$. A typical example class of profit functions satisfying (Hf) is given by : $f_{i}(x)=k_{i} x^{\gamma_{i}}, k_{i} \geq 0, i \in \mathbb{I}_{d}$, $0<\gamma_{1}<\ldots \gamma_{d} \leq 1$

In view of the ordering condition (Hf) on the regimes, it is natural to assume that the switching cost for an access to a higher regime is positive, i.e.

$$
(\mathbf{H g}+) \quad g_{i j}>0 \quad \text { for } i<j \in \mathbb{I}_{d} .
$$

We shall also assume that one receives some compensation when one switches to a lower regime, i.e.

$$
(\mathbf{H g}-) \quad g_{i j}<0 \quad \text { for } j<i \in \mathbb{I}_{d} .
$$

Notice that these conditions together with (2.9) imply the following ordering condition on the switching costs

$$
\begin{gathered}
0<g_{i j}<g_{i k} \quad \text { for } i<j<k \quad \in \mathbb{I}_{d} \\
0<-g_{i j}<-g_{i k} \quad \text { for } k<j<i \quad \in \mathbb{I}_{d} .
\end{gathered}
$$


In other words, the higher regime one wants to reach, the more one has to pay; and the lower regime one reaches, the more one receives.

Given a regime $i \in \mathbb{I}_{d}$, the first question is to determine under which conditions the switching region $\mathcal{S}_{i}$ is nonempty. Specifically, for $x \in \mathcal{S}_{i}$, one would like to know if one jumps to a higher regime, i.e. $x \in \mathcal{S}_{i j}$ for some $j>i$, or if one switches to a lower regime, i.e. $x \in \mathcal{S}_{i j}$ for $j<i$. So we introduce for any $i \in \mathbb{I}_{d}$ the upward and downward switching regions

$$
\mathcal{S}_{i}^{+}=\cup_{j>i} \mathcal{S}_{i j}, \quad \mathcal{S}_{i}^{-}=\cup_{j<i} \mathcal{S}_{i j},
$$

with the convention that $\mathcal{S}_{i}^{+}=\emptyset$ for $i=d$ and $\mathcal{S}_{i}^{-}=\emptyset$ for $i=1$. By definition, we have $\mathcal{S}_{i}=\mathcal{S}_{i}^{+} \cup \mathcal{S}_{i}^{-}$.

Remark 4.1 From the ordering condition (Hf), we have for all $j>i, Q_{i j}=\left[x_{i j}, \infty\right)$ for certain $x_{i j}>0$. Symmetrically, we have for all $j<i, Q_{i j}=\left(0, y_{i j}\right]$ for some $y_{i j}>0$. Together with Lemma 4.1, we get :

$$
\begin{aligned}
& \left(f_{i}-f_{j}\right)(x)+r g_{i j} \leq 0, \quad \forall j>i, \quad \forall x_{0} \in \mathcal{S}_{i j}, \quad \forall x \geq x_{0} \\
& \left(f_{i}-f_{j}\right)(x)+r g_{i j} \leq 0, \quad \forall j<i, \quad \forall y_{0} \in \mathcal{S}_{i j}, \quad \forall x \leq y_{0}
\end{aligned}
$$

We also have

$$
\mathcal{S}_{i}^{+} \subset \cup_{j>i} Q_{i j}=\left[x_{i}, \infty\right), \quad \mathcal{S}_{i}^{-} \subset \cup_{j<i} Q_{i j}=\left(0, y_{i}\right]
$$

for some $x_{i} \in(0, \infty], y_{i} \in(0, \infty)$. In particular,

$$
\begin{aligned}
0 & <\inf \mathcal{S}_{i}^{+} \leq \inf \mathcal{S}_{i j}, \quad \forall 1 \leq i<j \leq d, \\
\sup \mathcal{S}_{i j} & \leq \sup \mathcal{S}_{i}^{-}<\infty, \quad \forall 1 \leq j<i \leq d .
\end{aligned}
$$

We give some properties on switching regions that do intersect. We introduce the following definitions.

Definition 4.1 Let $i, j \in \mathbb{I}_{d}, j \neq i$, and $x_{0} \in \mathcal{S}_{i j}$. We say that $x_{0}$ is a left-boundary (resp. right-boundary) of $\mathcal{S}_{i j}$ if there exists some $\varepsilon>0$ s.t. $\left[x_{0}, x_{0}+\varepsilon\right)\left(\right.$ resp. $\left.\left(x_{0}-\varepsilon, x_{0}\right]\right) \subset \mathcal{S}_{i j}$ and $\left(x_{0}-\varepsilon, x_{0}\right) \cap \mathcal{S}_{i j}\left(\right.$ resp. $\left.\left(x_{0}, x_{0}+\varepsilon\right) \cap \mathcal{S}_{i j}\right)=\emptyset$.

Definition 4.2 Let $i, j, k \in \mathbb{I}_{d}, j \neq i, k \neq i, j \neq k$, and $x_{0} \in \mathcal{S}_{i j} \cap \mathcal{S}_{i k}$.

- $x_{0}$ is a crossing boundary point if it is a left-boundary of $\mathcal{S}_{i j}$ (resp. $\left.\mathcal{S}_{i k}\right)$ and a rightboundary of $\mathcal{S}_{i k}\left(\right.$ resp. $\left.\mathcal{S}_{i j}\right)$.

- $x_{0}$ is a $j$-isolated point if there exists some $\varepsilon>0$ s.t. $\left(x_{0}-\varepsilon, x_{0}\right]\left(\right.$ resp. $\left.\left[x_{0}, x_{0}+\varepsilon\right)\right)$ $\subset \mathcal{S}_{i k}$, and $\left(x_{0}-\varepsilon, x_{0}+\varepsilon\right) \cap \mathcal{S}_{i j}=\left\{x_{0}\right\}$.

Remark 4.2 Let $i, j \in \mathbb{I}_{d}, j \neq i$, with $\mathcal{S}_{i j}$ a singleton. Then, by Remark $3.3, \mathcal{S}_{i j}$ is reduced to a single $j$-isolated point. 
Lemma 4.2 For any $i, j, k \in \mathbb{I}_{d}, j \neq i, k \neq i, j \neq k$, we have $\operatorname{int}\left(\mathcal{S}_{i j} \cap \mathcal{S}_{i k}\right)=\emptyset$. Therefore, $\mathcal{S}_{i j} \cap \mathcal{S}_{i k}$ consists of only isolated or crossing boundaries points.

Proof. We argue by contradiction, and assume on the contrary that there exist some $i, j, k$ $\in \mathbb{I}_{d}, j \neq i, k \neq i, j \neq k, x_{0} \in \mathcal{S}_{i j} \cap \mathcal{S}_{i k}$, and $\varepsilon>0$ such that $\left(x_{0}-\varepsilon, x_{0}+\varepsilon\right) \subset \mathcal{S}_{i j} \cap \mathcal{S}_{i k}$. Since $\mathcal{S}_{i j}$ is included in the open continuation region $\mathcal{C}_{j}$ on which $v_{j}$ is $C^{2}$, we deduce that $v_{i}$ is $C^{2}$ on $\left(x_{0}-\varepsilon, x_{0}+\varepsilon\right)$. Recalling that $r v_{j}-\mathcal{L} v_{j}-f_{j}=0$ on $\mathcal{C}_{j}$, and since $v_{i}=v_{j}-g_{i j}$ on $\left(x_{0}-\varepsilon, x_{0}+\varepsilon\right) \subset \mathcal{C}_{j}$, we deduce that

$$
\begin{aligned}
r v_{i}(x)-\mathcal{L} v_{i}(x) & =r\left(v_{j}(x)-g_{i j}\right)-\mathcal{L} v_{j}(x) \\
& =f_{j}(x)-r g_{i j}, \quad \forall x \in\left(x_{0}-\varepsilon, x_{0}+\varepsilon\right) .
\end{aligned}
$$

Similarly, we have

$$
r v_{i}(x)-\mathcal{L} v_{i}(x)=f_{k}(x)-r g_{i k}, \quad \forall x \in\left(x_{0}-\varepsilon, x_{0}+\varepsilon\right) .
$$

By comparing the two previous equalities, we obtain

$$
\left(f_{j}-f_{k}\right)(x)=r\left(g_{i j}-g_{i k}\right), \quad \forall x \in\left(x_{0}-\varepsilon, x_{0}+\varepsilon\right),
$$

which is an obvious contradiction with (Hf).

Lemma 4.3 Let $x_{0} \in \mathcal{S}_{i j} \cap \mathcal{S}_{i k}, i, j, k \in \mathbb{I}_{d}, j \neq i, k \neq i, j \neq k$.

- If $x_{0}$ is a $j$-isolated point, then $\left(f_{k}-f_{j}\right)\left(x_{0}\right) \leq r\left(g_{i k}-g_{i j}\right)$.

- If $x_{0}$ is a crossing boundary point, then $\left(f_{k}-f_{j}\right)\left(x_{0}\right)=r\left(g_{i k}-g_{i j}\right)$.

Proof. Suppose that $x_{0}$ is a $j$-isolated point, i.e. w.l.o.g. there exists some $\varepsilon>0$ s.t. $\left(x_{0}-\varepsilon, x_{0}\right]\left(\right.$ resp. $\left.\left[x_{0}, x_{0}+\varepsilon\right)\right) \subset \mathcal{S}_{i k}$, and $\left(x_{0}-\varepsilon, x_{0}+\varepsilon\right) \cap \mathcal{S}_{i j}=\left\{x_{0}\right\}$. We set $G=$ $\left(v_{j}-g_{i j}\right)-\left(v_{k}-g_{i k}\right)$. Since $\mathcal{S}_{i j}$ (resp. $\left.\mathcal{S}_{i k}\right)$ is included in the open continuation set $\mathcal{C}_{j}$ (resp. $\mathcal{C}_{k}$ ) where $v_{j}$ (resp. $v_{k}$ ) satisfies $r v_{j}-\mathcal{L} v_{j}-f_{j}=0$ (resp. $r v_{k}-\mathcal{L} v_{k}-f_{k}=0$ ), we deduce that $G$ is $C^{2}$ in a neighborhood of $x_{0}$ and satisfies :

$$
r G-\mathcal{L} G=f_{j}-f_{k}-r\left(g_{i j}-g_{i k}\right) \quad \text { on }\left(x_{0}-\delta, x_{0}+\delta\right)
$$

for some $0<\delta<\varepsilon$. By definition of the switching regions, we have $v_{i}=v_{k}-g_{i k}>$ $v_{j}-g_{i j}$ on $\left(x_{0}, x_{0}+\varepsilon\right)$ with equality at $x_{0}$. By the smooth-fit property of $v_{i}$ at $x_{0}$, this implies : $G\left(x_{0}\right)=G^{\prime}\left(x_{0}\right)=0$, and $G^{\prime \prime}\left(x_{0}\right) \leq 0$. By sending $x$ to $x_{0}$ into (4.8), it follows that $\left(f_{j}-f_{k}\right)\left(x_{0}\right)-r\left(g_{i j}-g_{i k}\right) \geq 0$.

Suppose now that $x_{0}$ is a crossing boundary point, and so w.l.o.g. there exists $\varepsilon>0$ s.t. $\left(x_{0}-\varepsilon, x_{0}\right] \subset \mathcal{S}_{i j},\left[x_{0}, x_{0}+\varepsilon\right) \subset \mathcal{S}_{i k}$, with $\left(x_{0}-\varepsilon, x_{0}\right) \cap \mathcal{S}_{i k}=\left(x_{0}, x_{0}+\varepsilon\right) \cap \mathcal{S}_{i j}=\emptyset$. By setting $G=\left(v_{j}-g_{i j}\right)-\left(v_{k}-g_{i k}\right)$, and by the same arguments as above, we have (4.8). Moreover, we have $v_{i}=v_{j}-g_{i j}>v_{k}-g_{i k}$ on $\left(x_{0}-\varepsilon, x_{0}\right)$, and $v_{i}=v_{k}-g_{i k}>v_{j}-g_{i j}$ on $\left(x_{0}, x_{0}+\varepsilon\right)$, with equality at $x_{0}$. By the smooth-fit property of $v_{i}$ at $x_{0}$, this implies : $G\left(x_{0}\right)=G^{\prime}\left(x_{0}\right)=0, G^{\prime \prime}\left(x_{0}\right) \geq 0, G^{\prime \prime}\left(x_{0}\right) \leq 0$, and so $G^{\prime \prime}\left(x_{0}\right)=0$. Similarly, by observing that $v_{i}=v_{k}-g_{i k}>v_{i}-g_{i j}$ on $\left(x_{0}, x_{0}+\varepsilon\right)$, with equality at $x_{0}$, we have $G^{\prime \prime}\left(x_{0}^{+}\right) \leq 0$. By sending $x$ to $x_{0}$ in (4.8), we obtain the equality $\left(f_{j}-f_{k}\right)\left(x_{0}\right)-r\left(g_{i j}-g_{i k}\right)=0$.

We now give some properties on switching regions that are separated by a continuation region. 
Lemma 4.4 Let $i \in \mathbb{I}_{d}$, and suppose $\left(x_{0}, y_{0}\right)$ is a nonempty open bounded interval included in the continuation region $\mathcal{C}_{i}$ with $x_{0} \in \mathcal{S}_{i j}$ and $y_{0} \in \mathcal{S}_{i k}$ for some $j \neq i$ and $k \neq i$.

1) If $j>i$, then $k>i$ and $\left(f_{k}-f_{i}\right)\left(x_{0}\right)<r g_{i k}$. In particular, $x_{0}<\inf \mathcal{S}_{i k}$ and $k \neq j$.

2) If $j<i$ and $k<i$, then $\left(f_{i}-f_{j}\right)\left(y_{0}\right)>-r g_{i j}$. In particular, $y_{0}>\sup \mathcal{S}_{i j}$ and $k \neq j$.

Proof. We consider the (nonnegative) continuous function : $G=v_{i}-\max \left(v_{j}-g_{i j}, v_{k}-g_{i k}\right)$. Since $r v_{i}-\mathcal{L} v_{i}-f_{i}=0$ on $\mathcal{C}_{i}$ and hence on $\left(x_{0}, y_{0}\right) \subset \mathcal{C}_{i}$, and $r v_{j}-\mathcal{L} v_{j}-f_{j} \geq 0, r v_{k}-\mathcal{L} v_{k}-f_{k}$ $\geq 0$ on $(0, \infty)$ (in the viscosity sense), we easily check that $v$ is a viscosity subsolution to :

$$
r G-\mathcal{L} G \leq \max \left(f_{i}-f_{j}+r g_{i j}, f_{i}-f_{k}+r g_{i k}\right) \quad \text { on } \quad\left(x_{0}, y_{0}\right) .
$$

1) Suppose that $j>i$. Since $x_{0} \in \mathcal{S}_{i j}$, we have by (4.4):

$$
\left(f_{i}-f_{j}\right)(x)+r g_{i j} \leq 0, \quad \forall x \geq x_{0} .
$$

We first show that $k>i$. If not, i.e. $k<i$, and since $y_{0} \in \mathcal{S}_{i k}$, we have by (4.5) $: f_{i}-f_{k}+r g_{i k}$ $\leq 0$ on $\left(0, y_{i k}\right)$ and in particular on $\left(x_{0}, y_{0}\right)$. Therefore, we get from (4.9):

$$
r G-\mathcal{L} G \leq 0 \quad \text { on }\left(x_{0}, y_{0}\right)
$$

Now, since $x_{0} \in \mathcal{S}_{i j}, y_{0} \in \mathcal{S}_{i k}$, we have $G\left(x_{0}\right)=G\left(y_{0}\right)=0$. By the standard maximum principle, $G$ should non-positive on $\left(x_{0}, y_{0}\right)$. This is in contradiction with the fact that $\left(x_{0}, y_{0}\right) \in \mathcal{C}_{i}$ on which $G$ is strictly positive. Hence, $k>i$. We now prove that $\left(f_{k}-f_{i}\right)\left(x_{0}\right)$ $<r g_{i k}$, i.e. $x_{0} \notin Q_{i k}$. If it is not true, and since $Q_{i k}$ is an interval in the form $\left[x_{i k}, \infty\right)$, we would have $\left(f_{k}-f_{i}\right)(x)-r g_{i k} \geq 0$ for all $x \geq x_{0}$, and hence (4.10). As above, this provides the required contradiction. Finally, since $\mathcal{S}_{i k} \subset Q_{i k}$, this proves that $x_{0}<\inf \mathcal{S}_{i k}$, and in particular $k \neq j$.

2) Suppose that $j<i$ and $k<i$. Recalling that $y_{0} \in \mathcal{S}_{i k}$, we have by (4.5):

$$
\left(f_{i}-f_{k}\right)(x)+r g_{i k} \leq 0, \quad \forall x \leq y_{0} .
$$

We now prove that $\left(f_{i}-f_{j}\right)\left(y_{0}\right)>-r g_{i j}$, i.e. $y_{0} \notin Q_{i j}$. If not, and since $Q_{i j}$ is an interval in the form $\left(0, y_{i j}\right]$, we would have $\left(f_{i}-f_{j}\right)(y) \leq-r g_{i j}$, for all $x \leq y_{0}$, and so holds (4.10). By the same arguments as in 1 ), we get the required contradiction.

\subsection{Analysis of upward switching region}

The main results of this paragraph provide a qualitative description of the upward switching regions.

Proposition 4.1 Let $i \in \mathbb{I}_{d}$.

1) The switching region $\mathcal{S}_{i}^{+}$is nonempty if and only if :

$$
\cup_{j>i} Q_{i j} \neq \emptyset \Longleftrightarrow \exists j>i, \quad\left(f_{j}-f_{i}\right)(\infty)>r g_{i j} .
$$

2) Suppose $\mathcal{S}_{i}^{+} \neq \emptyset$. Then there exists a unique $j=j^{+}(i)>i$ such that $\sup \mathcal{S}_{i}^{+}=\sup \mathcal{S}_{i j}$ $=\infty$, and we have $\sup \mathcal{S}_{i k}<\infty$ for all $k>i, k \neq j^{+}(i)$. Moreover, $\mathcal{S}_{i j^{+}(i)}$ contains an interval in the form $\left[x_{i j^{+}(i)}, \infty\right)$ for some $x_{i j^{+}(i)} \in(0, \infty)$, and $j^{+}(i)=\min J(i)$ where

$$
J(i)=\left\{j \in \mathbb{I}_{d}, j>i:\left(f_{k}-f_{j}\right)(\infty) \leq r\left(g_{i k}-g_{i j}\right), \forall k \in \mathbb{I}_{d}, k>i\right\} .
$$


Economic interpretation. The first assertion gives explicit necessary and sufficient conditions under which, in a given regime, it is optimal to switch up. It means that one has interest to switch up if and only if one may find some higher regime so that the maximal net difference between the profit functions covers strictly the cost for a change of the regime. The interpretation of the second assertion is the following. In a given regime, say $i$, where one has interest to switch up (under the conditions of assertion 1), there is a unique regime where one should switch up to when the state is sufficiently large. Moreover, this uniquely chosen regime is explicitly determined as the minimum of the explicitly given set $J(i)$. In the two-regime case, i.e. $d=2$, we obviously have $j^{+}(1)=2$. In the multi-regime case, it can be practically calculated as follows :

- one first tests if $j_{1}=i+1$ lies in $J(i)$ i.e. if

$$
\left(P_{j_{1}}\right) \quad\left(f_{k}-f_{j_{1}}\right)(\infty) \leq r\left(g_{i k}-g_{i j_{1}}\right), \quad \forall k>j_{1}
$$

is satisfied. If yes, then $j^{+}(i)=j_{1}$.

- Otherwise, we denote

$$
j_{2}=\min \left\{k>j_{1}:\left(f_{k}-f_{j_{1}}\right)(\infty)>r\left(g_{i k}-g_{i j_{1}}\right)\right\},
$$

and we notice that $j \notin J(i)$ for $j_{1}<j<j_{2}$. Indeed, by definition of $j_{2}$, if $j<j_{2}$, then $\left(f_{j}-f_{j_{1}}\right)(\infty) \leq r\left(g_{i j}-g_{i j_{1}}\right)$ and so $\left(f_{j_{2}}-f_{j}\right)(\infty)=\left(f_{j_{2}}-f_{j_{1}}\right)(\infty)+\left(f_{j_{1}}-f_{j}\right)(\infty)>$ $r\left(g_{i j_{2}}-g_{i j_{1}}\right)+r\left(g_{i j_{1}}-g_{i j}\right)=r\left(g_{i j_{2}}-g_{i j}\right)$, or $j \notin J(i)$. Then, one tests if $j_{2}$ lies in $J(i)$. By observing that for $k<j_{2}$, we have $\left(f_{k}-f_{j_{2}}\right)(\infty)=\left(f_{k}-f_{j_{1}}\right)(\infty)+\left(f_{j_{1}}-f_{j_{2}}\right)(\infty)<$ $r\left(g_{i k}-g_{i j_{1}}\right)+r\left(g_{i j_{1}}-g_{i j_{2}}\right)=r\left(g_{i k}-g_{i j_{2}}\right)$, the test $j_{2} \in J(i)$ is written as :

$$
\left(P_{j_{2}}\right) \quad\left(f_{k}-f_{j_{1}}\right)(\infty) \leq r\left(g_{i k}-g_{i j_{1}}\right), \quad \forall k>j_{2} .
$$

If $\left(P_{j_{2}}\right)$ is satisfied, then $j^{+}(i)=j_{2}$.

- We continue by forward induction : if $\left(P_{j_{l}}\right)$ is satisfied, then $j^{+}(i)=j_{l}$. Otherwise, we denote

$$
j_{l+1}=\min \left\{k>j_{l}:\left(f_{k}-f_{j_{l}}\right)(\infty)>r\left(g_{i k}-g_{i j_{l}}\right)\right\}
$$

and one tests if $j_{l+1} \in J(i)$, i.e., if

$$
\left(P_{j_{l+1}}\right) \quad\left(f_{k}-f_{j_{l}}\right)(\infty) \leq r\left(g_{i k}-g_{i j_{l}}\right), \quad \forall k>j_{l}
$$

is satisfied. The property $\left(P_{j_{k}}\right)$ may be never satisfied in which case $j^{+}(i)=d$. For example, for the reward profit functions $f_{i}(x)=k_{i} x^{\gamma_{i}}$ with $k_{i}>0$ and $0<\gamma_{1}<\ldots \gamma_{d} \leq 1$, we have $\left(f_{k}-f_{j}\right)(\infty)=\infty$ for all $k>j$; and so $j^{+}(i)=d$.

\section{Proof of Proposition 4.1}

1) Notice first that the equivalence in (4.11) follows immediately from the definition of $Q_{i j}$ and the ordering condition (Hf). The necessary condition $\mathcal{S}_{i}^{+} \neq \emptyset \Longrightarrow \cup_{j>i} Q_{i j} \neq \emptyset$ is a direct consequence of the inclusion in Lemma 4.1. We now prove the following implication :

$$
\sup \mathcal{S}_{i}^{+}<\infty \Longrightarrow\left(f_{j}-f_{i}\right)(\infty) \leq r g_{i j}, \quad \forall j \in \mathbb{I}_{d}
$$

Indeed, assume that $\sup \mathcal{S}_{i}^{+}<\infty$. Then from (4.7), the switching region $\mathcal{S}_{i}$ is bounded. This means that the continuation region $\mathcal{C}_{i}$ contains $\left(y_{i}, \infty\right)$ for some $y_{i}>0$, and so $r v_{i}(x)-$ 
$\mathcal{L} v_{i}(x)-f_{i}(x)=0$ for $x>y_{i}$. Then, on $\left(y_{i}, \infty\right), v_{i}$ is in the form $: v_{i}(x)=\hat{V}_{i}(x)+A \psi^{-}(x)+$ $B \psi^{+}(x)$, for some constants $A$ and $B$. Moreover, from the linear growth condition on $v_{i}$ and condition (3.8), we must have $B=0$. Hence, recalling that $v_{i} \geq v_{j}-g_{i j}$ and $v_{j} \geq \hat{V}_{j}$ for all $j$, we have

$$
\hat{V}_{i}(x)+A \psi^{-}(x) \geq \hat{V}_{j}(x)-g_{i j}, \quad \forall x \geq y_{i} .
$$

By sending $x$ to infinity and from (2.6), (3.17), we obtain (4.13). This shows in particular that if $S_{i}^{+}=\emptyset$ (which obviously implies $\left.\sup \mathcal{S}_{i}^{+}<\infty\right)$, then $\cup_{j>i} Q_{i j}=\emptyset$. The proof of the equivalence in the first assertion is thus completed.

2) Now, suppose that $\mathcal{S}_{i}^{+} \neq \emptyset$, which means equivalently that (4.11) is satisfied. Hence, from (4.13), we must have $\sup \mathcal{S}_{i}^{+}=\infty$. Let us then consider the nonempty set $J_{\infty}(i)=$ $\left\{j>i: \sup \mathcal{S}_{i j}=\infty\right\}$.

Step 1. Fix some $j \in J_{\infty}(i)$ and let us prove that $\mathcal{S}_{i j}$ contains an interval in the form $\left[x_{i j}, \infty\right)$ for some $x_{i j}>0$. Since $\sup \mathcal{S}_{i j}=\infty$, one can find an increasing sequence $\left(x_{n}\right)_{n}$ in $\mathcal{S}_{i j}$ such that $\lim _{n} x_{n}=\infty$. We claim that there exists some $n$ such that $\mathcal{S}_{i j}$ contains the interval $\left[x_{n}, \infty\right)$. If not, then recalling that the switching regions are closed, one of the two following cases should occur for all $n$ :

- Case 1. there exists a non empty interval $\left(\tilde{x}_{n}, \tilde{y}_{n}\right)$ included in the continuation region $\mathcal{C}_{i}$, with $x_{n} \leq \tilde{x}_{n} \in \mathcal{S}_{i j^{\prime}}, \tilde{y}_{n} \in \mathcal{S}_{i k}$ for some $j^{\prime}, k \neq i$.

- Case 2. There exist some $k \neq j$, and an increasing sequence $\left(y_{n}^{m}\right)_{m}$ with $x_{n} \leq y_{n}^{0}, \lim _{m} y_{n}^{m}$ $=\infty$ such that $\mathcal{S}_{i j}$ contains $\cup_{m}\left[y_{n}^{2 m}, y_{n}^{2 m+1}\right]$ and $\mathcal{S}_{i k}$ contains $\cup_{m}\left[y_{n}^{2 \phi(m)+1}, y_{n}^{2 \phi(m)+2}\right]$, where $\phi$ is an increasing function valued in $\mathbb{N}$.

Since $\lim _{n} x_{n}=\infty$, we may assume, by taking $n$ large enough, that in Case $1 \tilde{x}_{n} \geq \inf \mathcal{S}_{i k}$. This, however, would contradict Lemma 4.41 ), and so Case 1 would never occur. We now show that Case 2 is also impossible. Indeed, in this case, recalling that $\mathcal{S}_{i j} \subset \mathcal{C}_{j}$, we have $\cup_{m \geq 0}\left[y_{n}^{2 m}, y_{n}^{2 m+1}\right] \subset \mathcal{C}_{j}$. We claim that $\mathcal{C}_{j}$ contains actually $\left[y_{n}^{m}, \infty\right)$ for some $n$ and $m$ large enough. Otherwise, for all $n, m$, one could find some nonempty interval $\left(z_{n}^{m}, \tilde{z}_{n}^{m}\right)$ included in $\mathcal{C}_{j}$, with $y_{n}^{m} \leq z_{n}^{m}<\tilde{z}_{n}^{m}$ and $z_{n}^{m} \in \mathcal{S}_{j k}, \tilde{z}_{n}^{m} \in \mathcal{S}_{j l}$ for some $k, l \neq j$. Since $\lim _{n} y_{n}^{m}=\infty$, we may assume, by taking $n$ large enough, that $z_{n}^{m} \geq \inf \mathcal{S}_{j l}$. This contradicts again Lemma 4.41 ), and hence $\left[y_{n}^{m}, \infty\right) \subset \mathcal{C}_{j}$ for some $m, n$. Fix such an $n$. Then, recalling that $\mathcal{S}_{i k}$ $\subset \mathcal{C}_{k}$ and $\lim _{m} y_{n}^{m}=\infty$, we show similarly that $\left[y_{n}^{m^{\prime}}, \infty\right) \subset \mathcal{C}_{k}$ for some $m^{\prime}$ large enough. By setting $m_{0}=m \vee m^{\prime}$, we then deduce that $\left[y_{n}^{m_{0}}, \infty\right)$ is included both in $\mathcal{C}_{j}$ and $\mathcal{C}_{k}$. Therefore, on $\left[y_{n}^{m_{0}}, \infty\right), v_{j}$ and $v_{k}$ satisfy respectively $r v_{j}-\mathcal{L} v_{j}-f_{j}=0$ and $r v_{k}-\mathcal{L} v_{k}-f_{k}$ $=0$. Since $v_{j}, v_{k}$ satisfy also the linear growth condition at infinity, they are in the form $v_{j}(x)=\hat{V}_{j}(x)+A_{j} \psi^{-}(x), v_{k}(x)=\hat{V}_{k}(x)+A_{k} \psi^{-}(x), x \in\left[y_{n}^{m_{0}}, \infty\right)$, for some constants $A_{j}$, $A_{k}$. Now, by writing that $y_{n}^{2 \phi(m)} \in \mathcal{S}_{i j} \cap \mathcal{S}_{i k}$, and by the very definition of the switching regions, we have for all $m \geq m_{0}$ :

$$
\begin{aligned}
v_{i}\left(y_{n}^{2 \phi(m)}\right) & =v_{j}\left(y_{n}^{2 \phi(m)}\right)-g_{i j}=\hat{V}_{j}\left(y_{n}^{2 \phi(m)}\right)+A_{j} \psi^{-}\left(y_{n}^{2 \phi(m)}\right)-g_{i j} \\
& =v_{k}\left(y_{n}^{2 \phi(m)}\right)-g_{i k}=\hat{V}_{k}\left(y_{n}^{2 \phi(m)}\right)+A_{k} \psi^{-}\left(y_{n}^{2 \phi(m)}\right)-g_{i k} .
\end{aligned}
$$

By sending $n$ to infinity into the r.h.s. of (4.14)-(4.15), and from (2.6), (3.17), we obtain :

$$
\left(f_{k}-f_{j}\right)(\infty)=r\left(g_{i k}-g_{i j}\right) .
$$


Since $k \neq j$, say, $k>j$, it follows from (Hf) that $\left(f_{k}-f_{j}\right)(x)<r\left(g_{i k}-g_{i j}\right)$ for all $x>0$. Consequently, in view of (3.15) we have

$$
\hat{V}_{k}(x)-\hat{V}_{j}(x)<g_{i k}-g_{i j}, \quad \forall x>0 .
$$

Hence, for all $x$ large enough, we have from (2.6)

$$
\hat{V}_{k}(x)+A_{k} \psi^{-}(x)-g_{i k}<\hat{V}_{j}(x)+A_{j} \psi^{-}(x)-g_{i j} .
$$

This contradicts (4.14)-(4.15) for $x=y_{n}^{2 \phi(m)}$, and so Case 2 never occurs.

Step 2. Fix some $j \in J_{\infty}(i)$. By the definition of the switching region and by Step 1, we have $v_{i}=v_{j}-g_{i j}$ on $\left[x_{i j}, \infty\right)$. Moreover, since $\mathcal{S}_{i j}$ is included in the continuation region $\mathcal{C}_{j}$, we deduce that $r v_{j}-\mathcal{L} v_{j}-f_{j}=0$ on $\left[x_{i j}, \infty\right)$; and so by the linear growth condition of $v_{j}$ at infinity, we have $v_{j}(x)=\hat{V}_{j}(x)+A_{j} \psi^{-}(x), x \geq x_{i j}$, for some constant $A_{j}$. Therefore,

$$
v_{i}(x)=\hat{V}_{j}(x)+A_{j} \psi^{-}(x)-g_{i j}, \quad \forall x \geq x_{i j} .
$$

Writing $v_{i} \geq v_{k}-g_{i k} \geq \hat{V}_{k}-g_{i k}$ for all $k$, we get :

$$
\hat{V}_{j}(x)+A \psi^{-}(x)-g_{i j} \geq \hat{V}_{k}(x)-g_{i j}, \quad \forall x \geq x_{i j}, \forall k \in \mathbb{I}_{d} .
$$

By sending $x$ to infinity and from (2.6), (3.17), we obtain

$$
\left(f_{j}-f_{k}\right)(\infty) \geq r\left(g_{i j}-g_{i k}\right), \quad \forall k \in \mathbb{I}_{d}
$$

This proves that $j$ lies in $J(i)$ defined in (4.12), and so $J_{\infty}(i) \subset J(i)$.

Step 3. We prove that $J_{\infty}(i)$ is reduced to a singleton. For this, consider $j, j^{\prime} \in J_{\infty}(i)$. From Step 2, there exists some $x_{i}^{\prime}>0$ such that

$$
v_{i}(x)=\hat{V}_{j}(x)+A_{j} \psi^{-}(x)-g_{i j}=\hat{V}_{j^{\prime}}(x)+A_{j^{\prime}} \psi^{-}(x)-g_{i j^{\prime}}, \quad \forall x \geq x_{i}^{\prime},
$$

for some constants $A_{j}, A_{j^{\prime}}$. Moreover, by writing that $j$ and $j^{\prime}$ lie in $J(i)$, we have :

$$
\left(f_{j^{\prime}}-f_{j}\right)(\infty)=r\left(g_{i j^{\prime}}-g_{i j}\right) .
$$

Thus, if we assume that $j^{\prime} \neq j$, we obtain a contradiction by the same argument as that in the end of Step 1.

Step 4. We finally prove that the singleton $J_{\infty}(i)$ consists of $j^{+}(i):=\min J(i)$. Let $j$ be the unique element in $J_{\infty}(i)$. Then, we recall from Step 1 that $v_{i}=\hat{V}_{j}+A_{j} \psi^{-}-g_{i j}$ on $\left[x_{i j}, \infty\right)$ for some $x_{i j}>0$ and constant $A_{j}$. Since $j, j_{+}(i) \in J(i)$, we have :

$$
\left(f_{j}-f_{j^{+}(i)}\right)(\infty)=r\left(g_{i j}-g_{i j^{+}(i)}\right),
$$

and obviously $j \geq j^{+}(i)$. Assume on the contrary that $j \neq j^{+}(i)$. Then $j>j^{+}(i)$, and by the same arguments as in (4.16), we obtain :

$$
\hat{V}_{j}(x)-\hat{V}_{j^{+}(i)}(x)<g_{i j}-g_{i j^{+}(i)}, \quad \forall x>0 .
$$

By (2.6), this implies for $x$ large enough :

$$
v_{i}(x)=\hat{V}_{j}+A_{j} \psi^{-}(x)-g_{i j}<\hat{V}_{j^{+}(i)}(x)-g_{i j^{+}(i)} \leq v_{j^{+}(i)}-g_{i j^{+}(i)} \leq v_{i}(x),
$$

a contradiction. 
Proposition 4.2 1) Let $i \in\{1, \ldots, d-1\}$ with $\mathcal{S}_{i}^{+} \neq \emptyset$. Suppose that

$$
\sup \left[\mathcal{S}_{i k} \backslash\left(\mathcal{S}_{i k} \cap \mathcal{S}_{i j^{+}(i)}\right)\right] \leq \inf \mathcal{S}_{i j^{+}(i)}, \quad \forall k \neq i, j^{+}(i) .
$$

Then, we have

$$
\mathcal{S}_{i j^{+}(i)}=\left[\bar{x}_{i j^{+}(i)}, \infty\right)
$$

with $\bar{x}_{i j^{+}(i)} \in(0, \infty)$.

2) Suppose that $\mathcal{S}_{1}^{+} \neq \emptyset$, and there exists $k \neq 1, j^{+}(1)$ such that $\mathcal{S}_{1 k}$ is nonempty and

$$
\sup \mathcal{S}_{1 k} \leq \inf \mathcal{S}_{1 j}, \quad \forall j \neq 1, k
$$

Then, $\mathcal{S}_{1 k}$ is in the form

$$
\mathcal{S}_{1 k}=\left[\bar{x}_{1 k}, \bar{y}_{1 k}\right]
$$

with $0<\bar{x}_{1 k} \leq \bar{y}_{1 k}<\infty$.

Proof. 1). We set $\bar{x}_{i j^{+}(i)}=\inf \mathcal{S}_{i j^{+}(i)}$, which is finite since $\mathcal{S}_{i j^{+}(i)}$ is nonempty (see Proposition 4.1). By (4.6), we also notice that $\bar{x}_{i j^{+}(i)}>0$. Suppose that (4.17) holds. Then, $\left(\bar{x}_{i j^{+}(i)}, \infty\right) \subset \mathcal{S}_{i j^{+}(i)} \cup \mathcal{C}_{i}$. From (3.3), we then deduce that $v_{i}$ is a viscosity solution to

$$
\min \left[r v_{i}-\mathcal{L} v_{i}-f_{i}, v_{i}-\left(v_{j^{+}(i)}-g_{i j^{+}(i)}\right)\right]=0 \quad \text { on } \quad\left(\bar{x}_{i j^{+}(i)}, \infty\right)
$$

Let us now consider the continuous function $w_{i}=v_{j^{+}(i)}-g_{i j^{+}(i)}$ on $\left[\bar{x}_{i j^{+}(i)}, \infty\right)$. We check that $w_{i}$ is a viscosity supersolution to

$$
r w_{i}-\mathcal{L} w_{i}-f_{i} \geq 0 \quad \text { on } \quad\left(\bar{x}_{i j^{+}(i)}, \infty\right)
$$

For this, take some point $\bar{x} \in\left(\bar{x}_{i j^{+}(i)}, \infty\right)$ and some smooth test function $\varphi$ s.t. $\bar{x}$ is a local minimum of $w_{i}-\varphi$. Then, $\bar{x}$ is a local minimum of $v_{j+(i)}-\left(\varphi+g_{i j^{+}(i)}\right)$. By writing the viscosity supersolution property of $v_{j^{+}(i)}$ to its Bellman PDE, we have :

$$
r v_{j^{+}(i)}(\bar{x})-\mathcal{L} \varphi(\bar{x})-f_{j^{+}(i)}(\bar{x}) \geq 0 .
$$

By applying inequality (4.4) to $\bar{x}>\bar{x}_{i j^{+}(i)} \in \mathcal{S}_{i j^{+}(i)}$, we have :

$$
\left(f_{j^{+}(i)}-f_{i}\right)(\bar{x})-r g_{i j^{+}(i)} \geq 0 .
$$

By adding these two last inequalities, we obtain the required supersolution inequality :

$$
r w_{i}(\bar{x})-\mathcal{L} \varphi(\bar{x})-f_{i}(\bar{x}) \geq 0,
$$

and so (4.20) is proved. Since $w_{i}=v_{j^{+}(i)}-g_{i j^{+}(i)}$, this proves that $w_{i}$ is a viscosity solution to :

$$
\min \left[r w_{i}-\mathcal{L} w_{i}-f_{i}, w_{i}-\left(v_{j^{+}(i)}-g_{i j^{+}(i)}\right)\right]=0 \quad \text { on } \quad\left(\bar{x}_{i j^{+}(i)}, \infty\right)
$$


Moreover, since $\bar{x}_{i j^{+}(i)} \in \mathcal{S}_{i j^{+}(i)}$, we have $w_{i}\left(\bar{x}_{i j^{+}(i)}\right)=v_{i}\left(\bar{x}_{i j^{+}(i)}\right)$. Observing also that $v_{i}$ and $w_{i}$ satisfy the linear growth condition at infinity, we deduce by uniqueness of viscosity solution to (4.19) that $v_{i}=w_{i}$ on $\left(\bar{x}_{i j^{+}(i)}, \infty\right)$, i.e. $\mathcal{S}_{i j^{+}(i)}=\left[\bar{x}_{i j^{+}(i)}, \infty\right)$.

2). We set $\bar{x}_{1 k}=\inf \mathcal{S}_{1 k}, \bar{y}_{1 k}=\sup \mathcal{S}_{1 k}$. By Proposition 4.12 ), we know that $\bar{y}_{1 k}<\infty$. From (4.6), we notice also that $\bar{x}_{1 k}>0$. Suppose that $\mathcal{S}_{1 k}$ is neither empty nor a singleton, so that $0<\bar{x}_{1 k}<\bar{y}_{1 k}<\infty$, and suppose that (4.18) holds. Let us prove that $\mathcal{S}_{1 k}=$ $\left[\bar{x}_{1 k}, \bar{y}_{1 k}\right]$. For this, we consider the function $w_{1}=v_{k}-g_{1 k}$ on $\left[\bar{x}_{1 k}, \bar{y}_{1 k}\right]$. The condition (4.18) implies that

$$
v_{1}(x)>v_{j}(x)-g_{1 j}, \quad \forall j \neq 1, k, \quad \forall x<\bar{y}_{1 k} .
$$

From (3.3), we deduce that $v_{1}$ is a viscosity solution to

$$
\min \left[r v_{1}-\mathcal{L} v_{1}-f_{1}, v_{1}-\left(v_{k}-g_{1 k}\right)\right]=0 \quad \text { on }\left(\bar{x}_{1 k}, \bar{y}_{1 k}\right) .
$$

By same arguments as in (4.20)-(4.21), we show that $w_{1}$ is a viscosity solution to

$$
\min \left[r w_{1}-\mathcal{L} w_{1}-f_{1}, w_{1}-\left(v_{k}-g_{1 k}\right)\right]=0 \quad \text { on }\left(\bar{x}_{1 k}, \bar{y}_{1 k}\right) .
$$

Moreover, since $\bar{x}_{1 k}$ and $\bar{y}_{1 k} \in \mathcal{S}_{1 k}$, we have $w_{1}\left(\bar{x}_{1 k}\right)=v_{1}\left(\bar{x}_{1 k}\right)$ and $w_{1}\left(\bar{y}_{1 k}\right)=v_{1}\left(\bar{y}_{1 k}\right)$. By uniqueness of viscosity solution to $(4.22)$, we deduce that $v_{1}=w_{1}$ on $\left[\bar{x}_{1 k}, \bar{y}_{1 k}\right]$, i.e. $\mathcal{S}_{1 k}=$ $\left[\bar{x}_{1 k}, \bar{y}_{1 k}\right]$.

Remark 4.3 In the two-regime case, i.e $d=2$, assertion 2) of the preceding proposition is not applicable while assertion 1) means that $\mathcal{S}_{1}=\mathcal{S}_{12}$ is either empty (see condition 1) in Proposition 4.1) or it is in the form $\left[\bar{x}_{12}, \infty\right)$ for some $\bar{x}_{12}>0$. Proposition 4.2 extends this result (already found in [17]) to the multi-regime case, and we shall see in particular in the next section how one can check conditions (4.17) and (4.18) in the three-regime case in order to determine the upward switching regions.

\subsection{Analysis of downward switching region}

The main results of this paragraph provide a qualitative description of the downward switching regions.

Proposition 4.3 For all $i=2, \ldots, d$, the switching region $\mathcal{S}_{i}^{-}$is nonempty. Moreover, $\inf \mathcal{S}_{i 1}=0, \mathcal{S}_{i 1}$ contains some interval in the form $\left(0, y_{i 1}\right], y_{i 1}>0$, and inf $\mathcal{S}_{i j}>0$ for all $1<j<i$.

Economic interpretation. The first assertion means that one always has interest to switch down due to the negative switching costs. Moreover, for small values of the state, one should switch down to the lowest regime $i=1$. This is intuitively justified by the fact that for small values of the state, the running profits are close to zero, and so one chooses the regime with the largest compensation fee, i.e. regime 1 , see (4.3).

\section{Proof of Proposition 4.3}


1. We first prove that $\inf \mathcal{S}_{i}^{-}=0$. If not, $\operatorname{since} \inf \mathcal{S}_{i}^{+}>0$ by (4.6), we then have inf $\mathcal{S}_{i}>0$. Therefore, the continuation region $\mathcal{C}_{i}$ contains $\left(0, y_{i}\right)$ for some $y_{i}>0$, and so $r v_{i}-\mathcal{L} v_{i}-f_{i}$ $=0$ on $\left(0, y_{i}\right)$. Then, on $\left(0, y_{i}\right), v_{i}$ is in the form $v_{i}(x)=\hat{V}_{i}(x)+A \psi^{-}(x)+B \psi^{+}(x)$, for some constants $A$ and $B$. Recalling that $v_{i}\left(0^{+}\right)$is finite, and under (2.6), we have $A=0$. By writing that $v_{i} \geq v_{j}-g_{i j} \geq \hat{V}_{j}-g_{i j}$ for all $j$, we have

$$
\hat{V}_{i}(x)+B \psi^{+}(x) \geq \hat{V}_{j}(x)-g_{i j}, \quad \forall x \in\left(0, y_{i}\right) .
$$

Sending $x$ to zero and from (2.6), we obtain $0 \geq-g_{i j}$. This is a contradiction with $(\mathbf{H g}-)$ for $j<i$. Therefore, $\inf \mathcal{S}_{i}^{-}=0$, and in particular $\mathcal{S}_{i}^{-} \neq \emptyset$.

2. Let us then consider the nonempty set $J_{0}(i)=\left\{j<i: \inf \mathcal{S}_{i j}=0\right\}$. Take some $j \in$ $J_{0}(i)$. Then, one can find a decreasing sequence $\left(x_{n}\right)_{n}$ in $\mathcal{S}_{i j} \operatorname{such}_{\text {that }} \lim _{n} x_{n}=0$. Since $\mathcal{S}_{i j}$ is closed, this implies that for $n$ large enough, $\mathcal{S}_{i j}$ contains the interval $\left(0, x_{n}\right]$. Then, $v_{i}=v_{j}-g_{i j}$ on $\left(0, x_{n}\right]$. Moreover, recalling that $\mathcal{S}_{i j}$ is included on $\mathcal{C}_{j}$, we deduce that $r v_{j}-\mathcal{L} v_{j}-f_{j}=0$ on $\left(0, x_{n}\right)$ and so $v_{i}=v_{j}-g_{i j}=\hat{V}_{j}+B \psi^{+}-g_{i j}$ on $\left(0, x_{n}\right)$. By writing that $v_{i} \geq v_{k}-g_{i k} \geq \hat{V}_{k}-g_{i k}$ for all $k$, we obtain :

$$
\hat{V}_{j}(x)+B \psi^{+}(x)-g_{i j} \geq \hat{V}_{k}(x)-g_{i k}, \quad \forall x \in\left(0, x_{n}\right) .
$$

By sending $x$ to zero, we conclude $-g_{i j} \geq-g_{i k}$ for all $k<i$. Under (4.3), this means that $j=1$, and the proof is completed.

Proposition 4.4 1) Let $i \in\{2, \ldots, d\}$, and suppose that

$$
\sup \mathcal{S}_{i 1} \leq \inf \left[\mathcal{S}_{i j} \backslash\left(\mathcal{S}_{i j} \cap \mathcal{S}_{i 1}\right)\right], \quad \forall j \neq 1, i
$$

Then, we have

$$
\mathcal{S}_{i 1}=\left(0, \underline{y}_{i 1}\right]
$$

with $\underline{y}_{i 1} \in(0, \infty)$.

2) Suppose there exists $k \neq 1, d$ such that $\mathcal{S}_{d k}$ is nonempty and

$$
\sup \mathcal{S}_{d j} \leq \inf \mathcal{S}_{d k}, \quad \forall j \neq d, k
$$

Then, $\mathcal{S}_{d k}$ is in the form

$$
\mathcal{S}_{d k}=\left[\underline{x}_{d k}, \underline{y}_{d k}\right]
$$

with $0<\underline{x}_{d k} \leq \underline{y}_{d k}<\infty$.

Proof. 1). We set $\underline{y}_{i 1}=\sup \mathcal{S}_{i 1}$, which is positive since $\mathcal{S}_{i 1}$ is nonempty (see Proposition 4.3). By (4.7), we also notice that $\underline{y}_{i 1}<\infty$. Suppose that (4.23) holds. Then, $\left(0, \underline{y}_{i 1}\right) \subset$ $\mathcal{S}_{i 1} \cup \mathcal{C}_{i}$. From (3.3), we deduce that $v_{i}$ is a viscosity solution to

$$
\min \left[r v_{i}-\mathcal{L} v_{i}-f_{i}, v_{i}-\left(v_{1}-g_{i 1}\right)\right]=0 \quad \text { on }\left(0, \underline{y}_{i 1}\right)
$$


Let us prove that $\mathcal{S}_{i 1}=\left(0, \underline{y}_{i 1}\right]$. To this end, we consider the function $w_{i}=v_{1}-g_{i 1}$ on $\left(0, \underline{y}_{i 1}\right]$. By same arguments as in (4.20)-(4.21), we show that $w_{i}$ is a viscosity solution to

$$
\min \left[r w_{i}-\mathcal{L} w_{i}-f_{i}, w_{i}-\left(v_{1}-g_{i 1}\right)\right]=0 \text { on }\left(0, \underline{y}_{i 1}\right) .
$$

Moreover, since $\inf \mathcal{S}_{i 1}=0$ and $\underline{y}_{i 1} \in \mathcal{S}_{i 1}$, we have $w_{i}\left(0^{+}\right)=v_{i}\left(0^{+}\right)\left(=-g_{i 1}\right)$ and $w_{i}\left(y_{i 1}\right)$ $=v_{i}\left(\underline{y}_{i 1}\right)$. By uniqueness of viscosity solution to (4.25), we deduce that $v_{i}=w_{i}$ on $\left(0, \underline{y}_{i 1}\right]$, i.e. $\mathcal{S}_{i 1}=\left(0, \underline{y}_{i 1}\right]$.

2). We set $\underline{x}_{d k}=\inf \mathcal{S}_{d k}, \underline{y}_{d k}=\sup \mathcal{S}_{d k}$. By Proposition 4.32 ), we know that $\underline{x}_{d k}>0$. From (4.7), we notice also that $\underline{y}_{d k}<\infty$. Suppose that $\mathcal{S}_{d k}$ is neither empty nor a singleton, so that $0<\underline{x}_{d k}<\underline{y}_{d k}<\infty$, and suppose that (4.24) holds. Let us prove that $\mathcal{S}_{d k}=\left[\underline{x}_{d k}, \underline{y}_{d k}\right]$. For this, we consider the function $w_{d}=v_{k}-g_{d k}$ on $\left[\underline{x}_{d k}, \underline{y}_{d k}\right]$. The condition (4.24) implies that

$$
v_{d}(x)>v_{j}(x)-g_{d j}, \quad \forall j \neq d, k, \quad \forall x>\underline{x}_{d k} .
$$

From (3.3), we deduce that $v_{d}$ is a viscosity solution to

$$
\min \left[r v_{d}-\mathcal{L} v_{d}-f_{d}, v_{d}-\left(v_{k}-g_{d k}\right)\right]=0 \quad \text { on } \quad\left(\underline{x}_{d k}, \underline{y}_{d k}\right) .
$$

By same arguments as in (4.20)-(4.21), we show that $w_{d}$ is a viscosity solution to

$$
\min \left[r w_{d}-\mathcal{L} w_{d}-f_{d}, w_{d}-\left(v_{k}-g_{d k}\right)\right]=0 \quad \text { on } \quad\left(\underline{x}_{d k}, \underline{y}_{d k}\right) .
$$

Moreover, since $\underline{x}_{d k}$ and $\underline{y}_{d k} \in \mathcal{S}_{d k}$, we have $w_{d}\left(\underline{x}_{d k}\right)=v_{d}\left(\underline{x}_{d k}\right)$ and $w_{d}\left(\underline{y}_{d k}\right)=v_{d}\left(\underline{y}_{d k}\right)$. By uniqueness of viscosity solution to $(4.26)$, we deduce that $v_{d}=w_{d}$ on $\left[\underline{x}_{d k}, \underline{y}_{d k}\right]$, i.e. $\mathcal{S}_{d k}=$ $\left[\underline{x}_{d k}, \underline{y}_{d k}\right]$.

Remark 4.4 In the two-regime case, i.e, $d=2$, assertion 2) of the preceding proposition does not apply while assertion 1$)$ means that $\mathcal{S}_{2}=\mathcal{S}_{21}$ is in the form $\left(0, \underline{y}_{21}\right]$ for some $\underline{y}_{21}$ $>0$. Proposition 4.4 extends this result (already found in [17]) to the multi-regime case, and we shall see in particular in the next section how one can check conditions (4.23) and (4.24) in the three-regime case in order to determine the downward switching regions.

\section{The three-regime case}

In this section, we consider the case of three regimes, i.e. $d=3$, and we show how one can use the results of the previous sections to obtain a fairly explicit description of the the switching regions.

We start with the lowest regime 1 . Notice that with regime 1 , we have $\mathcal{S}_{1}=\mathcal{S}_{1}^{+}=\mathcal{S}_{12}$ $\cup \mathcal{S}_{13}$

Theorem 5.1 (Switching regions in Regime 1)

1) Suppose that $\left(f_{2}-f_{1}\right)(\infty) \leq r g_{12}$ and $\left(f_{3}-f_{1}\right)(\infty) \leq r g_{13}$. Then $\mathcal{S}_{1}^{+}=\emptyset$.

2) Suppose that $\left(f_{2}-f_{1}\right)(\infty)>r g_{12}$ or $\left(f_{3}-f_{1}\right)(\infty)>r g_{13}$.

a) If $\left(f_{3}-f_{2}\right)(\infty) \leq r\left(g_{13}-g_{12}\right)$, then $\mathcal{S}_{12}=\left[\bar{x}_{12}, \infty\right)$ for some $\bar{x}_{12}>0$, and $\mathcal{S}_{13}=\emptyset$,

b) If $\left(f_{3}-f_{2}\right)(\infty)>r\left(g_{13}-g_{12}\right)$, then $\mathcal{S}_{13}=\left[\bar{x}_{13}, \infty\right)$ for some $\bar{x}_{13}>0$. Moreover, $\mathcal{S}_{12}$ is either empty or in the form $\mathcal{S}_{12}=\left[\bar{x}_{12}, \bar{y}_{12}\right]$ for some $0<\bar{x}_{12} \leq \bar{y}_{12} \leq \bar{x}_{13}$. 
Remark 5.1 The above qualitative description of the switching regions is explicit, depending only on the model parameters, in case 1) and 2a). For example, case 2a) means that when the maximal difference between profit functions in regime 3 and regime 2 is smaller than the difference between the corresponding switching costs, where it is never optimal to switch to regime 2, while it is optimal to wait until the state value reaches some threshold when one has interest to switch to regime 3 . In case $2 \mathrm{~b}$ ), the description is fairly explicit, although we are not able to give an explicit characterization when $\mathcal{S}_{12}$ is empty. We have an obvious necessary condition $\left(f_{2}-f_{1}\right)(\infty) \leq r g_{12}$, which implies under (Hf) that $Q_{12}=$ $\emptyset$ and so by Lemma $4.1, \mathcal{S}_{12}=\emptyset$. When the maximal difference between profit functions in regime 3 and regime 2 is greater than the difference between the corresponding switching costs, and $\left(f_{2}-f_{1}\right)(\infty)>r g_{12}$, we may have interest to switch to regime 2 for intermediate state values, while for large values of the state, one switches to regime 3 .

\section{Proof of Theorem 5.1}

1) Suppose first that $\left(f_{2}-f_{1}\right)(\infty) \leq r g_{12}$ and $\left(f_{3}-f_{1}\right)(\infty) \leq r g_{13}$. This is equivalent in view of Proposition 4.11$)$ to $\mathcal{S}_{1}=\mathcal{S}_{1}^{+}=\emptyset$. So $\mathcal{C}_{1}=(0, \infty), v_{1}=\hat{V}_{1}$; i.e., one always stays in regime 1.

2) Suppose now that $\left(f_{2}-f_{1}\right)(\infty)>r g_{12}$ or $\left(f_{3}-f_{1}\right)(\infty)>r g_{13}$. Equivalently, $\mathcal{S}_{1}=\mathcal{S}_{1}^{+}$ $\neq \emptyset$. We distinguish two subcases :

a) $\left(f_{3}-f_{2}\right)(\infty) \leq r\left(g_{13}-g_{12}\right)$.

By Proposition 4.1, we have $j^{+}(1)=2$, and $\bar{y}_{13}:=\sup \mathcal{S}_{13}<\infty$.

Step a1). Let us prove that $\mathcal{S}_{13}=\emptyset$. First, we claim that $\bar{y}_{13}<\bar{x}_{12}:=\inf \mathcal{S}_{12}$. We argue by contradiction by assuming $\bar{y}_{13} \geq \bar{x}_{12}$. Recalling from Proposition 4.12 ) that $\mathcal{S}_{12}$ contains an interval in the form $\left[x_{12}, \infty\right)$, together with Lemma 4.2 , we have the following two possible cases:

- $\bar{y}_{13}$ is a crossing boundary or 3-isolated point of $\mathcal{S}_{12} \cap \mathcal{S}_{13}$.

In this case, we have by Lemma 4.3 that $\left(f_{2}-f_{3}\right)\left(\bar{y}_{13}\right) \leq r\left(g_{12}-g_{13}\right)$. This would imply under $(\mathbf{H f})$ that $\left(f_{3}-f_{2}\right)(\infty)>r\left(g_{13}-g_{12}\right)$, a contradiction to the assumption of case $2 a)$.

- there exists $x_{12}>\bar{y}_{13}, x_{12} \in \mathcal{S}_{12}$ such that $\left(\bar{y}_{13}, x_{12}\right) \subset \mathcal{C}_{1}$.

In this case, it follows from Lemma 4.41 ) that $\bar{y}_{13}<\bar{x}_{12}$, a contradiction.

Hence, $\bar{y}_{13}<\bar{x}_{12}$ and $\left(\bar{y}_{13}, \bar{x}_{12}\right) \subset \mathcal{C}_{1}$. Let us now check that $\bar{y}_{13}=0$, i.e. $\mathcal{S}_{13}=\emptyset$. Otherwise, $\bar{y}_{13} \in \mathcal{S}_{13}$, and we have by $(4.4):\left(f_{3}-f_{1}\right)\left(\bar{y}_{13}\right) \geq r g_{13}$. Moreover, in view of the assumption of case $2 \mathrm{a})$ as well as under $(\mathbf{H f})$ that $f_{3}-f_{2} \leq r\left(g_{13}-g_{12}\right)$ on $(0, \infty)$, we deduce that

$$
\begin{aligned}
\left(f_{2}-f_{1}\right)\left(\bar{y}_{13}\right) & =\left(f_{2}-f_{3}\right)\left(\bar{y}_{13}\right)+\left(f_{3}-f_{1}\right)\left(\bar{y}_{13}\right) \\
& \geq r\left(g_{12}-g_{13}\right)+r g_{13}=r g_{12},
\end{aligned}
$$

a contradiction to Lemma 4.41 ). Therefore, $\mathcal{S}_{13}=\emptyset$.

Step a2). Since $\sup \mathcal{S}_{13}=0$, condition (4.17) is trivially satisfied with $i=1, j^{+}(i)=2$, and we deduce from Proposition 4.21$)$ that $\mathcal{S}_{1}=\mathcal{S}_{12}$ is in the form $\mathcal{S}_{12}=\left[\bar{x}_{12}, \infty\right)$ for some $\bar{x}_{12} \in(0, \infty)$. 
b) $\left(f_{3}-f_{2}\right)(\infty)>r\left(g_{13}-g_{12}\right)$.

By Proposition 4.1, we have $j^{+}(1)=3$.

Step b1). We claim that $\mathcal{S}_{13}$ is in the form $\mathcal{S}_{1}=\left[\bar{x}_{13}, \infty\right)$ for some $\bar{x}_{13} \in(0, \infty)$. We already know from Proposition 4.12 ) that $\mathcal{S}_{13}$ contains an interval in the form $\left[x_{13}, \infty\right)$. We then set $\bar{x}_{13}=\inf \left\{x_{0} \in \mathcal{S}_{13}:\left[x_{0}, \infty\right) \subset \mathcal{S}_{13}\right.$, and $\left.\exists x \notin \mathcal{S}_{13}, \quad x<x_{0}\right\}$. It suffices then to show that $\bar{x}_{13}=\inf \mathcal{S}_{13}$. We argue by contradiction by assuming on the contrary that $\inf \mathcal{S}_{13}<\bar{x}_{13}$. Recall that inf $\mathcal{S}_{13}>0$ by (4.6). Then, two possible cases could occur :

- there exists $z_{0} \in\left[\inf \mathcal{S}_{13}, \bar{x}_{13}\right), z_{0} \in \mathcal{S}_{1 j}$ for some $j \in\{2,3\}$ s.t. $\left(z_{0}, \bar{x}_{13}\right) \subset \mathcal{C}_{1}$.

By Lemma 4.41 ), we should have $z_{0}<\inf \mathcal{S}_{13}$, a contradiction.

- $\bar{x}_{13}$ is a crossing boundary point of $\mathcal{S}_{12} \cap \mathcal{S}_{13}$.

In this case, we must have by Lemma 4.3 :

$$
\left(f_{3}-f_{2}\right)\left(\bar{x}_{13}\right)=r\left(g_{13}-g_{12}\right) .
$$

We denote $y_{0}=\inf \left\{0<x_{0}<\bar{x}_{13}:\left[x_{0}, \bar{x}_{13}\right] \subset \mathcal{S}_{12}\right\}$, and we notice that $y_{0}<\bar{x}_{13}$ since $\bar{x}_{13}$ is a crossing boundary point of $\mathcal{S}_{12} \cap \mathcal{S}_{13}$. If $y_{0} \leq \inf \mathcal{S}_{13}$, then inf $\mathcal{S}_{13}$ would be a 3-isolated point of $\mathcal{S}_{12} \cap \mathcal{S}_{13}$ by Lemma 4.2 , and so by Lemma $4.3,\left(f_{2}-f_{3}\right)\left(\inf \mathcal{S}_{13}\right)$ $\leq r\left(g_{12}-g_{13}\right)$. Recalling that $\inf \mathcal{S}_{13}<\bar{x}_{13}$, this is in contradiction with (5.1) under (Hf). If, on the other hand, $y_{0}>\inf \mathcal{S}_{13}$, then we have two possibilities :

$\star\left(\inf \mathcal{S}_{13}, y_{0}\right)$ is included in $\mathcal{C}_{1}$. Since $\inf \mathcal{S}_{13} \in \mathcal{S}_{13}$, we have by $(4.4):\left(f_{3}-f_{1}\right)\left(\inf \mathcal{S}_{13}\right)$ $\geq r g_{13}$. Moreover, from (5.1) and under (Hf), we have $\left(f_{3}-f_{2}\right)(x) \leq r\left(g_{13}-g_{12}\right)$ for all $x \leq \bar{x}_{13}$, so that

$$
\begin{aligned}
\left(f_{2}-f_{1}\right)\left(\inf \mathcal{S}_{13}\right) & =\left(f_{2}-f_{3}\right)\left(\inf \mathcal{S}_{13}\right)+\left(f_{3}-f_{1}\right)\left(\inf \mathcal{S}_{13}\right) \\
\leq & r\left(g_{12}-g_{13}\right)+r g_{13}=r g_{12} .
\end{aligned}
$$

This contradicts Lemma 4.4 1).

$\star y_{0}$ is a crossing boundary of $\mathcal{S}_{12} \cap \mathcal{S}_{13}$. By Lemma (4.3), this would imply

$$
\left(f_{3}-f_{2}\right)\left(y_{0}\right)=r\left(g_{13}-g_{12}\right),
$$

a contradiction with (5.1) under (Hf), since $y_{0}<\bar{x}_{13}$.

We have thus proved that $\mathcal{S}_{13}=\left[\bar{x}_{13}, \infty\right)$ with $\bar{x}_{13}=\inf \mathcal{S}_{13}>0$.

Step b2. Let us now study the structure of $\mathcal{S}_{12}$. We suppose that $\mathcal{S}_{12}$ is neither empty, nor a singleton, and set $\bar{x}_{12}=\inf \mathcal{S}_{12}<\bar{y}_{12}=\sup \mathcal{S}_{12}$. Recall that $\bar{x}_{12}>0$ from (4.6). We also know from Proposition 4.12 ) that $\bar{y}_{12}<\infty$. Let us first show that $\bar{y}_{12} \leq \bar{x}_{13}$. If it is not true, then by Lemma 4.2 and since $\mathcal{S}_{13}=\left[\bar{x}_{13}, \infty\right), \bar{y}_{12}$ is a 2-isolated point of $\mathcal{S}_{12} \cap \mathcal{S}_{13}$, and one can find some $y_{0} \in \mathcal{S}_{12} y_{0}<\bar{y}_{12}$ such that $\left(y_{0}, \bar{y}_{12}\right) \cap \mathcal{S}_{12}=\emptyset$. By Lemma 4.3, $\left(f_{3}-f_{2}\right)\left(\bar{y}_{12}\right) \leq r\left(g_{13}-g_{12}\right)$, and so under (Hf), we have :

$$
\left(f_{3}-f_{2}\right)(x) \leq r\left(g_{13}-g_{12}\right), \quad \forall x<\bar{y}_{12} .
$$


Let us then consider the function $G=v_{1}-\left(v_{2}-g_{12}\right)$. Since $\left(y_{0}, \bar{y}_{12}\right) \cap \mathcal{S}_{12}=\emptyset$, i.e. $\left(y_{0}, \bar{y}_{12}\right)$ $\subset \mathcal{C}_{1} \cup \mathcal{S}_{13}$, we notice that $G$ is strictly positive on $\left(y_{0}, \bar{y}_{12}\right)$. Moreover, $v_{1}$ satisfies $r v_{1}-\mathcal{L} v_{1}$ $=f_{1}$ on $\mathcal{C}_{1}, v_{1}=v_{3}-g_{13}$ on $\mathcal{S}_{13} \subset \mathcal{C}_{3}$ satisfies : $r v_{1}-\mathcal{L} v_{1}=f_{3}-r g_{13}$, and $v_{2}$ satisfies $r v_{2}-\mathcal{L} v_{2}-f_{2} \geq 0$ on $(0, \infty)$. Hence, we deduce that $G$ is a viscosity subsolution to:

$$
r G-\mathcal{L} G \leq \max \left(f_{1}-f_{2}+r g_{12}, f_{3}-f_{2}+r\left(g_{12}-g_{13}\right)\right) \quad \text { on }\left(y_{0}, \bar{y}_{12}\right) .
$$

Since $\bar{x}_{12} \in \mathcal{S}_{12}$, we have by (4.4) :

$$
\left(f_{1}-f_{2}\right)(x)+r g_{12} \leq 0, \quad \forall x \geq \bar{x}_{12} .
$$

Together with (5.3), we obtain

$$
r G-\mathcal{L} G \leq 0 \quad \text { on }\left(y_{0}, \bar{y}_{12}\right) .
$$

Now, since $y_{0}$ and $\bar{y}_{12}$ lie in $\mathcal{S}_{12}$, we have $G\left(y_{0}\right)=G\left(\bar{y}_{12}\right)=0$. From the maximum principle, $G$ should be nonpositive on $\left(y_{0}, \bar{y}_{12}\right)$, a contradiction. Hence, $\bar{y}_{12}=\sup \mathcal{S}_{12} \leq \bar{x}_{13}$ $=\inf \mathcal{S}_{13}$. Condition (4.18) is then satisfied, and we deduce from Proposition 4.22 ) that $\mathcal{S}_{12}=\left[\bar{x}_{12}, \bar{y}_{12}\right]$.

We now provide an explicit qualitative description of the switching regions for the intermediate regime 2 . Notice that with regime 2 , we have $\mathcal{S}_{2}^{+}=\mathcal{S}_{23}$ and $\mathcal{S}_{2}^{-}=\mathcal{S}_{21}$.

Theorem 5.2 (Switching regions in Regime 2)

1) Suppose that $\left(f_{3}-f_{2}\right)(\infty) \leq r g_{23}$. Then $\mathcal{S}_{2}^{+}=\emptyset$, and $\mathcal{S}_{2}^{-}=\left(0, \underline{y}_{21}\right]$ for some $\underline{y}_{21}>0$.

2) Suppose that $\left(f_{3}-f_{2}\right)(\infty)>r g_{23}$. Then, $\mathcal{S}_{2}^{+}=\left[\bar{x}_{23}, \infty\right)$ and $\mathcal{S}_{2}^{-}=\left(0, \underline{y}_{21}\right]$ for some 0 $<\underline{y}_{21} \leq \bar{x}_{23}<\infty$.

Remark 5.2 The above qualitative description of the switching regions for regime 2 is explicit. When the maximal difference between the profit functions in the highest regime 3 and the current intermediate regime 2 is not large enough for covering the cost of changing of regime, one never switches up. However, if this maximal difference is larger than the corresponding switching cost, then one has interest to switch up starting from a certain threshold in state. On the other hand, one always switches down to the lowest regime 1 once the state value goes below a certain threshold. Moreover, the upward and downward switching regions may intersect only at some crossing boundary.

\section{Proof of Theorem 5.2}

1) Suppose that $\left(f_{3}-f_{2}\right)(\infty) \leq r g_{23}$, which means, in view of Proposition 4.11 ), that $\mathcal{S}_{2}^{+}$ $=\mathcal{S}_{23}=\emptyset$, and so inf $\mathcal{S}_{23}=\infty$. Condition (4.23) is then trivially satisfied with $i=2$, and we deduce from Proposition 4.41$)$ that $\mathcal{S}_{2}^{-}=\mathcal{S}_{21}=\left(0, \underline{y}_{21}\right]$ with $\underline{y}_{21} \in(0, \infty)$.

2) Suppose that $\left(f_{3}-f_{2}\right)(\infty)>r g_{23}$.

Step A. We first claim that $\mathcal{S}_{23}$ is in the form $\left[\bar{x}_{23}, \infty\right)$ for some $\bar{x}_{23}>0$. By Proposition $4.1, \mathcal{S}_{2}^{+}=\mathcal{S}_{23}$ is nonempty and contains an interval in the form $\left[x_{23}, \infty\right)$. We then set $\bar{x}_{23}$ $=\inf \left\{x_{0} \in \mathcal{S}_{23}:\left[x_{0}, \infty\right) \subset \mathcal{S}_{23}\right.$, and $\left.\exists x \notin \mathcal{S}_{23}, \quad x<x_{0}\right\}$. It suffices then to show that $\bar{x}_{23}=\inf \mathcal{S}_{23}$. We argue by contradiction by assuming on the contrary that inf $\mathcal{S}_{23}<\bar{x}_{23}$. Then, two possible cases could occur : 
- there exists $z_{0} \in\left[\inf \mathcal{S}_{23}, \bar{x}_{23}\right), z_{0} \in \mathcal{S}_{2 j}$ for some $j \in\{1,3\}$ s.t. $\left(z_{0}, \bar{x}_{23}\right) \subset \mathcal{C}_{2}$.

In this case, by Lemma 4.41 ), we must have $j=1$ and $z_{0}>\inf \mathcal{S}_{23}$. Moreover, by using again Lemma 4.4 between $\inf \mathcal{S}_{23}$ and $z_{0}$, we deduce that $\mathcal{S}_{23}$ should intersect with $\mathcal{S}_{21}$ at some crossing boundary point $\xi_{0}$ with $\left[\inf \mathcal{S}_{23}, \xi_{0}\right] \subset \mathcal{S}_{23}$ and $\left[\xi_{0}, z_{0}\right] \subset$ $\mathcal{S}_{21}$. By Lemma 4.3 , we have $\left(f_{3}-f_{1}\right)\left(\xi_{0}\right)=r\left(g_{23}-g_{21}\right)$ and so under (Hf) :

$$
\left(f_{1}-f_{3}\right)(x)+r\left(g_{23}-g_{21}\right) \leq 0, \quad \forall x \geq \xi_{0} .
$$

By (4.4), we have

$$
\left(f_{2}-f_{3}\right)(x)+r g_{23} \leq 0, \quad \forall x \geq \inf \mathcal{S}_{23}
$$

Let us consider the continuous function $G=v_{2}-\left(v_{3}-g_{23}\right)$ on $\left[\xi_{0}, \bar{x}_{23}\right]$. Since $v_{2}=$ $v_{1}-g_{21}$ on $\left(\xi_{0}, z_{0}\right) \subset \mathcal{C}_{1}$ satisfies : $r v_{2}-\mathcal{L} v_{2}=f_{1}-r g_{21}, v_{2}$ satisfies on $\left(z_{0}, \bar{x}_{23}\right) \subset$ $\mathcal{C}_{2}: r v_{2}-\mathcal{L} v_{2}=f_{2}$, and $r v_{3}-\mathcal{L} v_{3}-f_{3} \geq 0$ on $(0, \infty)$, we deduce that $G$ is a viscosity subsolution to :

$$
\begin{aligned}
r G-\mathcal{L} G & \leq \max \left[f_{1}-f_{3}+r\left(g_{23}-g_{21}\right), f_{2}-f_{3}+r g_{23}\right] \\
& \leq 0 \text { on }\left(\xi_{0}, \bar{x}_{i j^{+}(i)}\right)
\end{aligned}
$$

from (5.6)-(5.7). Since $G\left(\xi_{0}\right)=G\left(\bar{x}_{23}\right)=0$, this implies by the standard maximum principle that $G$ is non-positive on $\left(\xi_{0}, \bar{x}_{i j^{+}(i)}\right)$, a contradiction.

- $\bar{x}_{23}$ is a crossing boundary point of $\mathcal{S}_{21} \cap \mathcal{S}_{23}$.

By the same arguments as those in proving Theorem 5.1 2b) (Step b1), we can show that this case is impossible.

We have then proved that $\mathcal{S}_{23}$ is actually equal to $\left[\bar{x}_{23}, \infty\right)$ with $\bar{x}_{23}=\inf \mathcal{S}_{23}$.

Step B. Let us now study the structure of the downward switching region $\mathcal{S}_{2}^{-}=\mathcal{S}_{21}$. From Proposition $4.3, \mathcal{S}_{21}$ is nonempty and contains an interval in the form $\left(0, y_{21}\right]$. We set $\underline{y}_{21}$ $:=\sup \mathcal{S}_{21}<\infty$ by (4.7). Let us show that $\underline{y}_{21} \leq \bar{x}_{23}$. If it is not true, then by Lemma 4.2 and since $\mathcal{S}_{23}=\left[\bar{x}_{23}, \infty\right), \underline{y}_{21}$ is a 1-isolated point of $\mathcal{S}_{21} \cap \mathcal{S}_{23}$, and one can find some $y_{0}$ $\in \mathcal{S}_{21}, y_{0}<\underline{y}_{21}$ s.t. $\left(y_{0}, \underline{y}_{21}\right) \cap \mathcal{S}_{21}=\emptyset$. By Lemma $4.3,\left(f_{3}-f_{1}\right)\left(\underline{y}_{21}\right) \leq r\left(g_{23}-g_{21}\right)$, and so under (Hf), we have :

$$
\left(f_{3}-f_{1}\right)(x)+r\left(g_{21}-g_{23}\right) \leq 0, \quad \forall x<\underline{y}_{21} .
$$

By (4.5), we also have

$$
\left(f_{2}-f_{1}\right)(x)+r g_{21} \leq 0, \quad \forall x \leq \underline{y}_{21} .
$$

Let us then consider the function $G=v_{2}-\left(v_{1}-g_{21}\right)$. Since $\left(y_{0}, \underline{y}_{21}\right) \cap \mathcal{S}_{21}=\emptyset$, i.e. $\left(y_{0}, \underline{y}_{21}\right)$ $\subset \mathcal{C}_{2} \cup \mathcal{S}_{23}$, we notice that $G$ is strictly positive on $\left(y_{0}, \underline{y}_{21}\right)$. Moreover, $v_{2}$ satisfies $r v_{2}-\mathcal{L} v_{2}$ $=f_{2}$ on $\mathcal{C}_{2}, v_{2}=v_{3}-g_{23}$ on $\mathcal{S}_{23} \subset \mathcal{C}_{3}$ satisfies : $r v_{2}-\mathcal{L} v_{2}=f_{3}-r g_{23}$, and $v_{1}$ satisfies $r v_{1}-\mathcal{L} v_{1}-f_{1} \geq 0$ on $(0, \infty)$. Hence, we deduce that $G$ is a viscosity subsolution to

$$
r G-\mathcal{L} G \leq \max \left(f_{2}-f_{1}+r g_{21}, f_{3}-f_{1}+r\left(g_{21}-g_{23}\right)\right) \leq 0 \quad \text { on }\left(y_{0}, \underline{y}_{21}\right),
$$


from (5.8)-(5.9). Since $G\left(y_{0}\right)=G\left(\underline{y}_{21}\right)=0$, this implies by the maximum principle that $G$ is non-positive on $\left(y_{0}, \underline{y}_{21}\right)$, a contradiction. Therefore, $\underline{y}_{21}=\sup \mathcal{S}_{21} \leq \bar{x}_{23}=\inf \mathcal{S}_{23}$. Condition (4.23) is then satisfied, and we deduce from Proposition 4.41$)$ that $\mathcal{S}_{21}=\left(0, \underline{y}_{21}\right]$.

We finally provide a quasi-explicit qualitative description of the switching regions in the highest regime 3 . Notice that with regime 3 we have $\mathcal{S}_{3}=\mathcal{S}_{3}^{-}=\mathcal{S}_{31} \cup \mathcal{S}_{32}$.

Theorem 5.3 (Switching regions in Regime 3)

We have $\mathcal{S}_{31}=\left(0, \underline{y}_{31}\right]$ for some $\underline{y}_{31}>0$. Moreover, $\mathcal{S}_{32}$ is either empty or in the form $\left[\underline{x}_{32}, \underline{y}_{32}\right]$ for some $\underline{y}_{31} \leq \underline{x}_{32} \leq \underline{y}_{32}<\infty$.

Remark 5.3 This theorem states that one always switches down to the lowest regime 1 once the state value goes below a certain threshold. Moreover, one may have also interest to switch down to the intermediate regime when the state value lies in some closed bounded interval, which intersects possibly with the switching region for regime 1 , but only at a crossing boundary.

\section{Proof of Theorem 5.3}

Step 1. By Proposition 4.3, we know that $\mathcal{S}_{31}$ contains an interval in the form $\left(0, y_{31}\right]$ for some $y_{31}>0$ and $\inf \mathcal{S}_{31}=0$. We set $\underline{y}_{31}=\sup \left\{y_{0} \in \mathcal{S}_{31}:\left(0, y_{0}\right] \subset \mathcal{S}_{31}\right.$, and $\left.\exists y \notin \mathcal{S}_{31}, y_{0}<y\right\}$. Let us show that $\underline{y}_{31}=\sup \mathcal{S}_{31}$ so that $\mathcal{S}_{31}=\left(0, \underline{y}_{31}\right)$. We argue by contradiction by assuming on the contrary that $\underline{y}_{31}<\sup \mathcal{S}_{31}$. Then, two possible cases could occur:

- Case 1: there exists $z_{0} \in\left(\underline{y}_{31}, \sup \mathcal{S}_{31}\right], z_{0} \in \mathcal{S}_{3 j}$ for some $j \in\{1,2\}$ such that $\left(\underline{y}_{31}, z_{0}\right)$ $\subset \mathcal{C}_{3}$.

In this case, by Lemma 4.42 ), we should have $z_{0}>\sup \mathcal{S}_{31}$, a contradiction.

- Case 2: $\underline{y}_{31}$ is a crossing boundary point of $\mathcal{S}_{31} \cap \mathcal{S}_{32}$.

In this case, we must have by Lemma 4.3 :

$$
\left(f_{2}-f_{1}\right)\left(\underline{y}_{31}\right)=r\left(g_{32}-g_{31}\right) \text {. }
$$

We denote $y_{0}=\sup \left\{\xi_{0}>\underline{y}_{31}:\left[\underline{y}_{31}, \xi_{0}\right] \subset \mathcal{S}_{32}\right\}$, and notice that $y_{0}>\underline{y}_{31}$ since $\underline{x}_{31}$ is a crossing boundary point of $\mathcal{S}_{31} \cap \mathcal{S}_{32}$. If $y_{0} \geq \sup \mathcal{S}_{31}$, then $\sup \mathcal{S}_{31}$ would be a 1-isolated point of $\mathcal{S}_{31} \cap \mathcal{S}_{32}$ by Lemma 4.2 , and so by Lemma $4.3,\left(f_{2}-f_{1}\right)\left(\sup \mathcal{S}_{31}\right)$ $\leq r\left(g_{32}-g_{21}\right)$. Recalling that $\sup \mathcal{S}_{31}>\underline{y}_{31}$, this contradicts (5.10) under (Hf). If $y_{0}<\sup \mathcal{S}_{31}$, then we have two possibilities :

$\star\left(y_{0}, \sup \mathcal{S}_{31}\right)$ is included in $\mathcal{C}_{3}$. By $(4.5)$, we have $\left(f_{3}-f_{1}\right)\left(\sup \mathcal{S}_{31}\right) \leq-r g_{31}$. Moreover, from (5.10) and under (Hf), we have $\left(f_{2}-f_{1}\right)(x) \geq r\left(g_{32}-g_{31}\right)$ for all $x \geq \underline{y}_{31}$, so that

$$
\begin{aligned}
\left(f_{3}-f_{2}\right)\left(\sup \mathcal{S}_{31}\right) & =\left(f_{3}-f_{1}\right)\left(\sup \mathcal{S}_{31}\right)+\left(f_{1}-f_{2}\right)\left(\sup \mathcal{S}_{31}\right) \\
& \leq-r g_{31}+r\left(g_{31}-g_{32}\right)=-r g_{32} .
\end{aligned}
$$


This is in contradiction with Lemma 4.42 ).

$\star y_{0}$ is a crossing boundary of $\mathcal{S}_{31} \cap \mathcal{S}_{32}$. By Lemma (4.3), this would imply

$$
\left(f_{2}-f_{1}\right)\left(y_{0}\right)=r\left(g_{32}-g_{31}\right),
$$

a contradiction with (5.10) under (Hf), since $y_{0}>\underline{y}_{31}$.

We have then proved that $\mathcal{S}_{31}=\left(0, \underline{y}_{31}\right]$ with $\underline{y}_{31}=\sup \mathcal{S}_{31}$.

Step 2. Let us now study the structure of $\mathcal{S}_{32}$. Suppose that $\mathcal{S}_{32}$ is neither empty, nor a singleton. We recall that $\underline{y}_{32}:=\sup \mathcal{S}_{32}<\infty$ by (4.7) and that $\underline{x}_{32}:=\inf \mathcal{S}_{32}>0$ thanks to Proposition 4.3. Let us first prove that $\underline{y}_{31} \leq \underline{x}_{32}$. If not, then by Lemma 4.2 and since $\mathcal{S}_{31}=\left(0, \underline{y}_{31}\right], \underline{x}_{32}$ is a 2 -isolated point of $\mathcal{S}_{31} \cap \mathcal{S}_{32}$, and one can find some $y_{0} \in \mathcal{S}_{32}, \underline{x}_{32}$ $<y_{0}$ such that $\left(\underline{x}_{32}, y_{0}\right) \cap \mathcal{S}_{32}=\emptyset$. By Lemma $4.3,\left(f_{1}-f_{2}\right)\left(\underline{x}_{32}\right) \leq r\left(g_{31}-g_{32}\right)$, and so under (Hf), we have :

$$
\left(f_{1}-f_{2}\right)(x)+r\left(g_{32}-g_{31}\right) \leq 0, \quad \forall x \geq \underline{x}_{32} .
$$

By (4.5), we also have

$$
\left(f_{3}-f_{2}\right)(x)+r g_{32} \leq 0, \quad \forall x \leq \underline{y}_{32} .
$$

Let us then consider the function $G=v_{3}-\left(v_{2}-g_{32}\right)$. Since $\left(\underline{x}_{32}, y_{0}\right) \cap \mathcal{S}_{32}=\emptyset$, i.e. $\left(\underline{x}_{32}, y_{0}\right)$ $\subset \mathcal{C}_{3} \cup \mathcal{S}_{31}$, we notice that $G$ is strictly positive on $\left(\underline{x}_{32}, y_{0}\right)$. Moreover, $v_{3}$ satisfies $r v_{3}-\mathcal{L} v_{3}$ $=f_{3}$ on $\mathcal{C}_{3}, v_{3}=v_{1}-g_{31}$ on $\mathcal{S}_{31} \subset \mathcal{C}_{1}$ satisfies : $r v_{3}-\mathcal{L} v_{3}=f_{1}-r g_{31}$, and $v_{2}$ satisfies $r v_{2}-\mathcal{L} v_{2}-f_{2} \geq 0$ on $(0, \infty)$. Hence, we deduce that $G$ is a viscosity subsolution to

$$
r G-\mathcal{L} G \leq \max \left(f_{3}-f_{2}+r g_{32}, f_{1}-f_{2}+r\left(g_{32}-g_{32}\right)\right) \leq 0 \quad \text { on }\left(\underline{x}_{32}, y_{0}\right)
$$

from (5.12)-(5.13). Since $G\left(\underline{x}_{32}\right)=G\left(y_{0}\right)=0$, this implies by the maximum principle that $G$ is non-positive on $\left(\underline{x}_{32}, y_{0}\right)$, a contradiction. Therefore, $\underline{y}_{31}=\sup \mathcal{S}_{31} \leq \underline{x}_{32}=\inf \mathcal{S}_{32}$. Condition (4.24) is then satisfied, and we conclude from Proposition 4.42 ) that $\mathcal{S}_{32}=$ $\left[\underline{x}_{32}, \underline{y}_{32}\right]$.

We finally summarize the qualitative structure of the switching regions in the threeregime model; see also Figure 1 for a visual aid.

Theorem 5.4 (Switching regions in the three-regime model)

We have the following four cases :

A) If $\left(f_{2}-f_{1}\right)(\infty) \leq r g_{12},\left(f_{3}-f_{1}\right)(\infty) \leq r g_{13}$, and $\left(f_{3}-f_{2}\right)(\infty) \leq r g_{23}$, then

$$
\begin{aligned}
& \mathcal{S}_{1}=\mathcal{S}_{1}^{+}=\emptyset, \\
& \mathcal{S}_{2}^{-}=\left(0, \underline{y}_{21}\right], \quad \mathcal{S}_{2}^{+}=\emptyset, \\
& \mathcal{S}_{31}=\left(0, \underline{y}_{31}\right], \quad \mathcal{S}_{32} \text { is either empty or } \mathcal{S}_{32}=\left[\underline{x}_{32}, \underline{y}_{32}\right]
\end{aligned}
$$

for some $0<\underline{y}_{31} \leq \underline{x}_{32} \leq \underline{y}_{32}<\infty, 0<\underline{y}_{21}<\underline{x}_{32}$. 
B) If $\left(f_{2}-f_{1}\right)(\infty)>r g_{12}$ or $\left(f_{3}-f_{1}\right)(\infty)>r g_{13}$, and $\left(f_{3}-f_{2}\right)(\infty) \leq r\left(g_{13}-g_{12}\right)$, then

$$
\begin{aligned}
\mathcal{S}_{12}=\left[\bar{x}_{12}, \infty\right), & \mathcal{S}_{13}=\emptyset, \\
\mathcal{S}_{2}^{-}=\left(0, \underline{y}_{21}\right], & \mathcal{S}_{2}^{+}=\emptyset, \\
\mathcal{S}_{31}=\left(0, \underline{y}_{31}\right], & \mathcal{S}_{32} \text { is either empty or } \mathcal{S}_{32}=\left[\underline{x}_{32}, \underline{y}_{32}\right]
\end{aligned}
$$

for some $0<\underline{y}_{21} \leq \bar{x}_{12}, 0<\underline{y}_{31} \leq \underline{x}_{32} \leq \underline{y}_{32}<\infty, \underline{y}_{31}<\bar{x}_{12}<\infty, 0<\underline{y}_{21}<\underline{x}_{32}$.

C) If $\left(f_{3}-f_{1}\right)(\infty)>r g_{13}$, and $r\left(g_{13}-g_{12}\right)<\left(f_{3}-f_{2}\right)(\infty) \leq r g_{23}$, then

$$
\begin{aligned}
\mathcal{S}_{13}=\left[\bar{x}_{13}, \infty\right), & \mathcal{S}_{12} \text { is either empty or } \mathcal{S}_{12}=\left[\bar{x}_{12}, \bar{y}_{12}\right] \\
\mathcal{S}_{2}^{-}=\left(0, \underline{y}_{21}\right], & \mathcal{S}_{2}^{+}=\emptyset, \\
\mathcal{S}_{31}=\left(0, \underline{y}_{31}\right], & \mathcal{S}_{32} \text { is either empty or } \mathcal{S}_{32}=\left[\underline{x}_{32}, \underline{y}_{32}\right],
\end{aligned}
$$

for some $0<\bar{x}_{12} \leq \bar{y}_{12} \leq \bar{x}_{13}<\infty, 0<\underline{y}_{31} \leq \underline{x}_{32} \leq \underline{y}_{32}<\infty, 0<\underline{y}_{21}<\bar{x}_{12}, \underline{y}_{31}<\underline{x}_{13}$. D) If $\left(f_{3}-f_{1}\right)(\infty)>r g_{13}$, and $\left(f_{3}-f_{2}\right)(\infty)>r g_{23}$, then

$$
\begin{aligned}
\mathcal{S}_{13}=\left[\bar{x}_{13}, \infty\right), & \mathcal{S}_{12} \text { is either empty or } \mathcal{S}_{12}=\left[\bar{x}_{12}, \bar{y}_{12}\right] \\
\mathcal{S}_{2}^{-}=\left(0, \underline{y}_{21}\right], & \mathcal{S}_{2}^{+}=\left[\bar{x}_{23}, \infty\right), \\
\mathcal{S}_{31}=\left(0, \underline{y}_{31}\right], & \mathcal{S}_{32} \text { is either empty or } \mathcal{S}_{32}=\left[\underline{x}_{32}, \underline{y}_{32}\right],
\end{aligned}
$$

for some $0<\bar{x}_{12} \leq \bar{y}_{12} \leq \bar{x}_{13}<\infty, 0<\underline{y}_{31} \leq \underline{x}_{32} \leq \underline{y}_{32}<\bar{x}_{23}<\infty, 0<\underline{y}_{21}<\bar{x}_{12}, \bar{y}_{12}$ $<\bar{x}_{23}, \underline{y}_{31}<\underline{x}_{13}$.

Proof. Assertion A) follows from Theorem 5.1 1), Theorem 5.2 1) and Theorem 5.3. Assertion B) follows from Theorem 5.1 2a), Theorem 5.2 1) and Theorem 5.3, and observing that $g_{13}-g_{12}<g_{23}$ from (2.10). Assertion C) follows from Theorem $5.12 \mathrm{~b}$ ), Theorem 5.2 1 ) and Theorem 5.3, and observing that $\left(f_{3}-f_{1}\right)(\infty)>r g_{13}$ when $\left(f_{2}-f_{1}\right)(\infty)>r g_{12}$ and $r\left(g_{13}-g_{12}\right)<\left(f_{3}-f_{2}\right)(\infty)$. Assertion D) follows from Theorem $\left.5.12 \mathrm{~b}\right)$, Theorem 5.22 ) and Theorem 5.3. Finally, the other ordering condition on the thresholds of the switching regions follows from the observation that a switching region $\mathcal{S}_{i j}$ is included in the continuation region $\mathcal{C}_{j}$, and hence never intersects with $\mathcal{S}_{j k}$.

\section{Numerical procedure}

The qualitative structure of optimal switching controls derived in the previous section states that the optimal sequence of stopping times is given by the hitting times of the diffusion process $X$ at a finite number of threshold levels. This is of vital importance in eventually solving (either analytically or numerically) our problem, because it reduces the originally very complex problem into one finding a small number of critical values in state which is a finite-dimensional optimization problem. In this section, we demonstrate how to design algorithms to find these critical values in state. We shall take case B) in Theorem 5.4 to showcase our approach. This is not unduly technical, yet it is rich enough to catch the essential feature and difficulty of such a problem. 


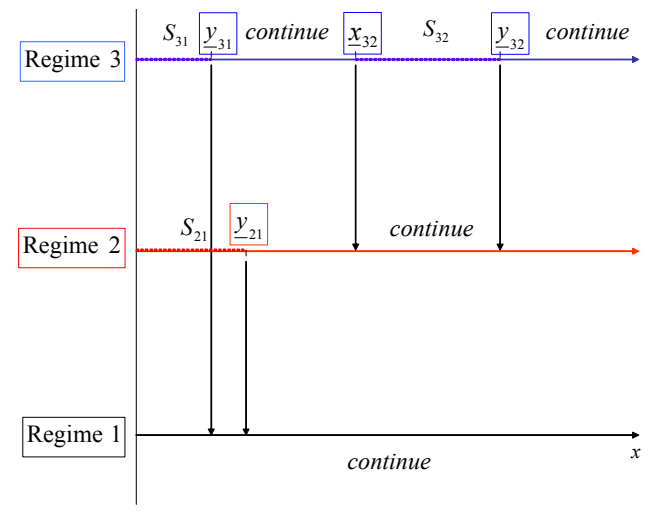

Case A

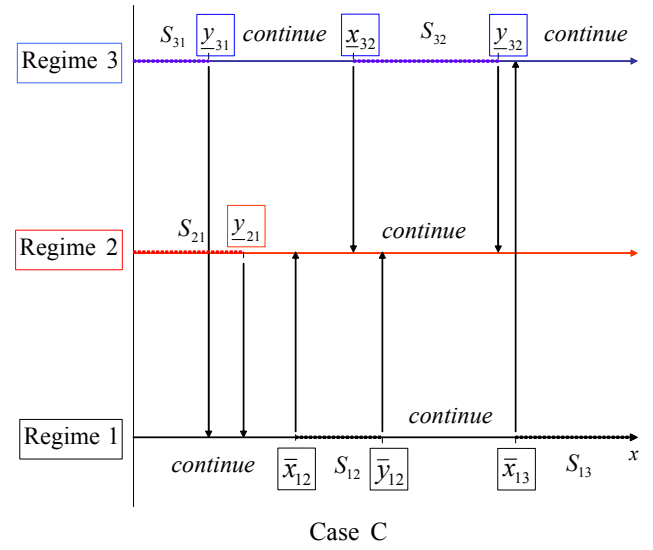

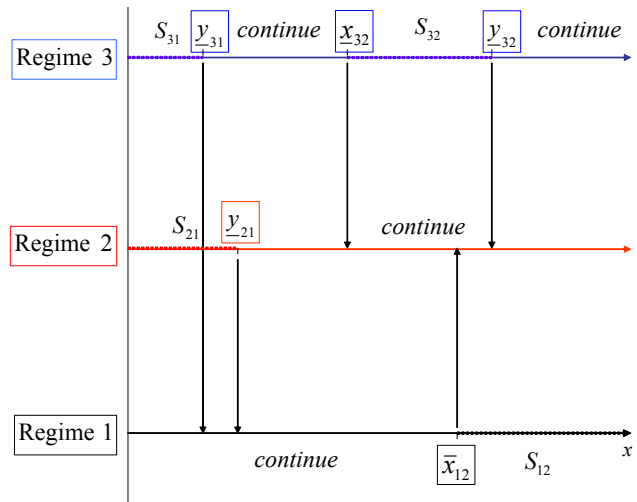

Case B

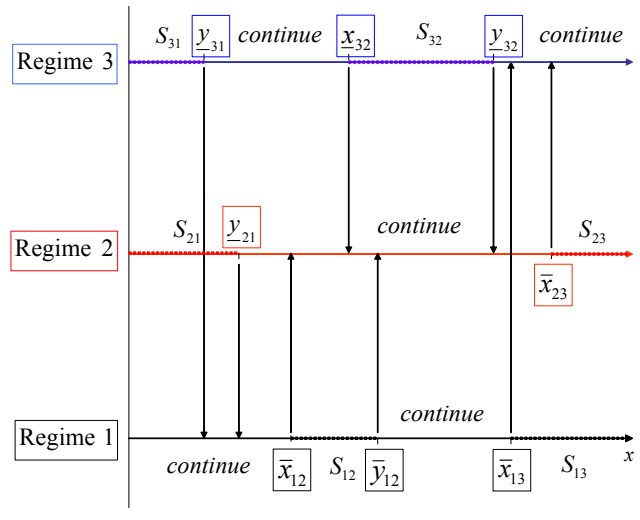

Case D

Figure 1: Switching regions in the three-regime model 
The optimal control of $X_{t}$ is completely dictated by the feedback law in Theorem 5.4, case B), except we do not yet know the values of the five threshold parameters : $\bar{x}_{12}, \underline{y}_{21}$, $\underline{y}_{31}, \underline{x}_{32}, \underline{y}_{32}$, which we are now to find. Notice that these parameters should satisfy :

$$
0<\underline{y}_{21} \leq \bar{x}_{12}, 0<\underline{y}_{31}<\bar{x}_{12}, 0<\underline{y}_{21}<\underline{x}_{32}, 0<\underline{y}_{31} \leq \underline{x}_{32}<\underline{y}_{32} .
$$

In accordance with our notation, we denote by $J_{i}(x)$ the total profit if one starts with regime $i(i=1,2,3)$ and state $x>0$. Bear in mind that $J_{i}(x)$ would depend on the values of the five threshold parameters, and that the value function $v_{i}(x)$ is the maximum of $J_{i}(x)$ over these parameters. Actually, we shall explicitly express $J_{i}(x)$ and $v_{i}(x)$ in terms of expectation functionals involving hitting times for the state process $X$, and derive a procedure to compute the optimal threshold parameters.

We first introduce some notations. For $x>0, a, b>0$, let

$$
\tau_{a}^{x}=\inf \left\{t \geq 0: \quad X_{t}^{x}=a\right\}, \quad \tau_{a b}^{x}=\inf \left\{t \geq \tau_{a}: X_{t}^{x}=b\right\}
$$

Fix $\rho>0$, and denote

$$
R(x, a)=E\left[e^{-\rho \tau_{a}^{x}}\right], \quad \hat{R}(x, a, b)=E\left[e^{-\rho \tau_{a b}^{x}}\right], \tilde{R}(x, a, b)=E\left[e^{-\rho \tau_{a}^{x}} 1_{\tau_{x}^{a}<\tau_{b}^{x}}\right] .
$$

For any measurable function $f$ on $\mathbb{R}_{+}$with linear growth condition, and for $x, a, b>0$, we also set

$$
\begin{aligned}
& F(f ; x, a)=E\left[\int_{0}^{\tau_{a}^{x}} e^{-\rho t} f\left(X_{t}^{x}\right) d t\right], \quad \hat{F}(f ; x, a, b)=E\left[\int_{\tau_{a}^{x}}^{\tau_{a b}^{x}} e^{-\rho t} f\left(X_{t}^{x}\right) d t\right], \\
& \tilde{F}(f ; x, a, b)=E\left[\int_{0}^{\tau_{a}^{x} \wedge \tau_{b}^{x}} e^{-\rho t} f\left(X_{t}^{x}\right) d t\right] .
\end{aligned}
$$

These expectation functionals $R, \hat{R}, \tilde{R}, F, \hat{F}$, and $\tilde{F}$, are calculated in closed analytic form when $X$ is a GBM (see Appendix), and may be approximated by Euler scheme discretization and Monte-Carlo simulations for general diffusion $X$.

We now turn to the computation of the value functions $v_{i}$ and the optimal threshold parameters. This is achieved in two steps.

\section{Step I : determination of threshold parameters and value functions in regimes 1 and 2}

We start with regime 1 . Fix some $x>0$, and let us compute $J_{1}(x)$ and $v_{1}(x)$. Notice that since starting from regime 1 we can only switch eventually to regime $2, J_{1}(x)$ depends only on the two threshold parameters $\bar{x}_{12}$ and $\underline{y}_{21}$. We stress this dependence by writing $J_{1}(x)=J_{1}\left(x, \bar{x}_{12}, \underline{y}_{21}\right)$, and calculate the function $J_{1}(x,$.$) on the domain \mathcal{D}_{12}=\left\{\left(\bar{x}_{12}, \underline{y}_{21}\right) \in\right.$ $\left.(0, \infty)^{2}: \underline{y}_{21} \leq \bar{x}_{12}\right\}$ as follows :

- If $\bar{x}_{12}>x$, then the optimal strategy is to let the process diffuse until it hits $\bar{x}_{12}$ at the stopping time $\tau_{\bar{x}_{12}}^{x}$ when one switches to regime 2 (and paying the cost $g_{12}$ ), and then lets it diffuse. One then switches back to regime 1 (paying the cost $g_{21}$ ) as soon 
as the process hits $\underline{y}_{21}$ at $\tau_{\bar{x}_{12} \underline{y}_{21}}^{x}$. After $\tau_{\bar{x}_{12} \underline{y}_{21}}^{x}$, the process repeats itself as described above. Specifically, we have for $\bar{x}_{12}>x$ :

$$
\begin{gathered}
J_{1}\left(x, \bar{x}_{12}, \underline{y}_{21}\right)=E\left[\int_{0}^{\tau_{\bar{x}_{12}}^{x}} e^{-\rho t} f_{1}\left(X_{t}^{x}\right) d t-e^{-\rho \tau_{\bar{x}_{12}}^{x}} g_{12}+\int_{\tau_{\bar{x}_{12}}^{x}}^{\tau_{\bar{x}_{12}}^{x} \underline{y}_{21}} e^{-\rho t} f_{2}\left(X_{t}^{x}\right) d t\right. \\
\left.+e^{-\rho \tau_{\bar{x}_{12}}^{x} \underline{y}_{21}}\left(-g_{21}+J_{1}\left(\underline{y}_{21}, \bar{x}_{12}, \underline{y}_{21}\right)\right)\right] \\
=F\left(f_{1} ; x, \bar{x}_{12}\right)-R\left(x, \bar{x}_{12}\right) g_{12}+\hat{F}\left(f_{2} ; x, \bar{x}_{12}, \underline{y}_{21}\right) \\
\quad+\hat{R}\left(x, \bar{x}_{12}, \underline{y}_{21}\right)\left(-g_{21}+J_{1}\left(\underline{y}_{21}, \bar{x}_{12}, \underline{y}_{21}\right)\right)
\end{gathered}
$$

Notice that this last relation for $x=\underline{y}_{21}$ yields the expression of $J_{1}\left(\underline{y}_{21}, \bar{x}_{12}, \underline{y}_{21}\right)$ by :

$$
\begin{aligned}
& \left(1-\hat{R}\left(\underline{y}_{21}, \bar{x}_{12}, \underline{y}_{21}\right)\right) J_{1}\left(\underline{y}_{21}, \bar{x}_{12}, \underline{y}_{21}\right) \\
= & F\left(f_{1} ; \underline{y}_{21}, \bar{x}_{12}\right)-R\left(\underline{y}_{21}, \bar{x}_{12}\right) g_{12}+\hat{F}\left(f_{2} ; \underline{y}_{21}, \bar{x}_{12}, \underline{y}_{21}\right)-\hat{R}\left(\underline{y}_{21}, \bar{x}_{12}, \underline{y}_{21}\right) g_{21}
\end{aligned}
$$

Hence, plugging the above into (6.4), we obtain an explicit expression of $J_{1}\left(x, \bar{x}_{12}, \underline{y}_{21}\right)$ for $\bar{x}_{12}>x$, in terms of the expectation functionals $R, \hat{R}, F$ and $\hat{F}$.

- If $\bar{x}_{12} \leq x$, then the optimal strategy is to switch immediately at time $t=0$ to regime 2 , and then as in the first case, let it diffuse until it hits $\underline{y}_{21}$ and so on. Similarly as above, we express $J_{1}\left(x, \bar{x}_{12}, \underline{y}_{21}\right)$ for $\bar{x}_{12} \leq x$ in terms of $R, \hat{R} F$ and $\hat{F}$ :

$$
\begin{aligned}
J_{1}\left(x, \bar{x}_{12}, \underline{y}_{21}\right) & =-g_{12}+E\left[\int_{0}^{\tau_{\underline{y}}^{x}} e^{-\rho t} f_{2}\left(X_{t}^{x}\right) d t+e^{-\rho \tau_{\underline{y}_{21}}^{x}}\left(-g_{21}+J_{1}\left(\underline{y}_{21}, \bar{x}_{12}, \underline{y}_{21}\right)\right)\right] \\
& =-g_{12}+F\left(f_{2}, x, \underline{y}_{21}\right)+R\left(x, \underline{y}_{21}\right)\left(-g_{21}+J_{1}\left(\underline{y}_{21}, \bar{x}_{12}, \underline{y}_{21}\right)\right) .
\end{aligned}
$$

The value $v_{1}(x)$, which is the maximum of the expected profit over the threshold parameters, is then determined by :

$$
v_{1}(x)=\max _{\left(\bar{x}_{12}, \underline{y}_{21}\right) \in \mathcal{D}_{12}} J_{1}\left(x, \bar{x}_{12}, \underline{y}_{21}\right),
$$

and the optimal threshold parameters $\bar{x}_{12}^{*}$ and $\underline{y}_{21}^{*}$ are the solutions to the above argmax optimization (which is trivially easy). Notice that the optimal threshold parameters do not depend on the current state value $x$; so once these have been calculated, the computation of $v_{1}\left(x^{\prime}\right)$ for other state values $x^{\prime}>0$, is directly derived from the relation :

$$
v_{1}\left(x^{\prime}\right)=J_{1}\left(x^{\prime}, \bar{x}_{12}^{*}, \underline{y}_{21}^{*}\right) .
$$

Moreover, the value function $v_{2}(x)$ for regime 2 is derived as follows :

- if $x>\underline{y}_{21}^{*}$, then the optimal strategy is to let the process diffuse until it hits $\underline{y}_{21}^{*}$ at the stopping time $\tau_{\underline{y}_{12}^{*}}^{x}$ when one switchs to regime 1 and finally follows the remaining optimal strategy computed above. Hence,

$$
\begin{aligned}
v_{2}(x) & =E\left[\int_{0}^{\tau_{21}^{x}} e^{-\rho t} f_{2}\left(X_{t}^{x}\right) d t+e^{-\rho \tau_{\underline{y}_{21}^{*}}^{x}}\left(-g_{21}+v_{1}\left(\underline{y}_{21}^{*}\right)\right)\right] \\
& =F\left(f_{2}, x, \underline{y}_{21}^{*}\right)+R\left(x, \underline{y}_{21}^{*}\right)\left(-g_{21}+v_{1}\left(\underline{y}_{21}^{*}\right)\right) .
\end{aligned}
$$


- $x \leq \underline{y}_{21}^{*}$, then the optimal strategy is to switch immediately to regime 1 . Hence,

$$
v_{2}(x)=-g_{21}+v_{1}(x)
$$

\section{Step II : determination of threshold parameters and value function in regime 3}

The optimal threshold parameters $\bar{x}_{12}^{*}, \underline{y}_{21}^{*}$ and the value functions $v_{1}$ and $v_{2}$ for regimes 1 and 2 are known from Step I, and we now move on to find the threshold parameters in regime $3: \underline{y}_{31}$, and $\underline{x}_{32}, \underline{y}_{32}$ if they exist, together with the value function $v_{3}(x)$. What is tricky here is that Theorem 5.4 does not decisively tell us whether the interval of switching from regime 3 to regime $2, \mathcal{S}_{32}=\left[\underline{x}_{32}, \underline{y}_{32}\right]$, exists. The approach presented here is able to determine such existence, by comparing the two options when starting from regime 3 : one is to let the process diffuse until it hits $\underline{y}_{31}$, and then follow the remaining optimal strategy, and the other is to switch to regime 2 , and then follow the optimal strategy afterwards. If the latter option is better off for some state value $x$, then it suggests that $\left[\underline{x}_{32}, \underline{y}_{32}\right]$ is nonempty. So, we start from regime 3 , fix some $x>0$, and consider the following two options :

- Option 1 : the interval $\left[\underline{x}_{32}, \underline{y}_{32}\right]$ does not exist. Denote $J_{3}^{(1)}\left(x, \underline{y}_{31}\right)$ the corresponding expected profit in regime 3 with threshold value $\underline{y}_{31}$. The function $\underline{y}_{31} \rightarrow J_{3}^{(1)}\left(x, \underline{y}_{31}\right)$ is calculated on the domain $\left(0, \bar{x}_{12}^{*}\right)$ as follows :

- If $\underline{y}_{31}<x$, then the optimal strategy would be to let the process diffuse until it hits $\underline{y}_{31}$ when one switchs to regime 1 , and then follow the remaining optimal strategy determined in Step I. Hence, we have for $\underline{y}_{31}<x$ :

$$
\begin{aligned}
J_{3}^{(1)}\left(x, \underline{y}_{31}\right) & =E\left[\int_{0}^{\tau_{\underline{x}_{31}}^{x}} e^{-\rho t} f_{3}\left(X_{t}^{x}\right) d t+e^{-\rho \tau_{\underline{y}_{31}}^{x}}\left(-g_{31}+v_{1}\left(\underline{y}_{31}\right)\right)\right] \\
& =F\left(f_{3}, x, \underline{y}_{31}\right)+R\left(x, \underline{y}_{31}\right)\left(-g_{31}+v_{1}\left(\underline{y}_{31}\right)\right) .
\end{aligned}
$$

- If $\underline{y}_{31} \geq x$, then the optimal strategy would be to switch immediately to regime 1 , and then follow the remaining optimal strategy determined in Step I. Hence, we have for $\underline{y}_{31} \geq x$ :

$$
J_{3}^{(1)}\left(x, \underline{y}_{31}\right)=-g_{31}+v_{1}(x)
$$

The maximal expected profit in Option 1 is then given by :

$$
v_{3}^{(1)}(x)=\sup _{\underline{y}_{31} \in\left(0, \bar{x}_{12}^{*}\right)} J_{3}^{(1)}\left(x, \underline{y}_{31}\right) .
$$

- Option 2 : the interval $\left[\underline{x}_{32}, \underline{y}_{32}\right]$ exists (possibly a singleton). Denote $J_{3}^{(2)}\left(x, \underline{y}_{31}, \underline{x}_{32}, \underline{y}_{32}\right)$ the corresponding expected profit in regime 3 with threshold parameters $\underline{y}_{31}, \underline{x}_{32}, \underline{y}_{32}$. The function $J_{3}^{(2)}(x,$.$) is calculated on the domain \mathcal{D}_{3}^{(2)}=\left\{\left(\underline{y}_{31}, \underline{x}_{32}, \underline{y}_{32}\right) \in(0, \infty)^{3}\right.$ : $\left.\underline{y}_{31}<\bar{x}_{12}^{*}, \underline{y}_{21}^{*}<\underline{x}_{32}, \underline{y}_{31} \leq \underline{x}_{32} \leq \underline{y}_{32}\right\}$ as follows : 
- If $x>\underline{y}_{32}$, then the optimal strategy is to let the process diffuse until it hits $\underline{y}_{32}$ when one switchs to regime 2 , and then follow the remaining optimal strategy determined in Step I. Hence, we have for $x>\underline{y}_{32}$ :

$$
\begin{aligned}
J_{3}^{(2)}\left(x, \underline{y}_{31}, \underline{x}_{32}, \underline{y}_{32}\right) & =E\left[\int_{0}^{\tau_{\underline{y}_{32}}^{x}} e^{-\rho t} f_{3}\left(X_{t}^{x}\right) d t+e^{-\rho \underline{y}_{32}^{x}}\left(-g_{32}+v_{2}\left(\underline{y}_{32}\right)\right)\right] \\
& =F\left(f_{3}, x, \underline{y}_{32}\right)+R\left(x, \underline{y}_{32}\right)\left(-g_{32}+v_{2}\left(\underline{y}_{32}\right)\right) .
\end{aligned}
$$

- If $\underline{x}_{32} \leq x \leq \underline{y}_{32}$, then the optimal strategy is to switch immediately to regime 2 , and then follow the remaining optimal strategy determined in Step I. Hence, we have for $\underline{x}_{32} \leq x \leq \underline{y}_{32}:$

$$
J_{3}^{(2)}\left(x, \underline{y}_{31}, \underline{x}_{32}, \underline{y}_{32}\right)=-g_{32}+v_{2}(x)
$$

- If $\underline{y}_{31}<x<\underline{x}_{32}$, then the optimal strategy is to let the process diffuse until it hits either $\underline{y}_{31}$ (when one switches to regime 1 ) or $\underline{x}_{32}$ (when one switches to regime 2), and then follow the remaining optimal strategy determined in Step I. Hence, we have for $\underline{y}_{31}<x<\underline{x}_{32}$ :

$$
\begin{aligned}
& J_{3}^{(2)}\left(x, \underline{y}_{31}, \underline{x}_{32}, \underline{y}_{32}\right)=E\left[\int_{0}^{\tau_{\underline{y}_{31}}^{x} \wedge \tau_{\underline{x}_{32}}^{x}} e^{-\rho t} f_{3}\left(X_{t}^{x}\right) d t\right. \\
& +e^{-\rho \tau_{\underline{y}_{31}}^{x}}\left(-g_{31}+v_{1}\left(\underline{y}_{31}\right)\right) 1_{\tau_{\underline{\underline{y}}_{31}}^{x}<\tau_{\underline{x}_{32}}^{x}} \\
& \left.+e^{-\rho{\underline{x_{32}}}_{32}^{x}}\left(-g_{32}+v_{2}\left(\underline{x}_{32}\right)\right) 1_{\tau_{\underline{x}_{32}}^{x}<\tau_{\underline{y}_{31}}^{x}}\right] \\
& =\tilde{F}\left(f_{3} ; \underline{y}_{31}, \underline{x}_{32}\right)+\tilde{R}\left(x, \underline{y}_{31}, \underline{x}_{32}\right)\left(-g_{31}+v_{1}\left(\underline{y}_{31}\right)\right) \\
& +\tilde{R}\left(x, \underline{x}_{32}, \underline{y}_{31}\right)\left(-g_{32}+v_{2}\left(\underline{x}_{32}\right)\right) \text {. }
\end{aligned}
$$

- If $\underline{y}_{31}=x=\underline{x}_{32}$, then the optimal strategy is to switch immediately to regime 1 or 2 , and to follow the remaining optimal strategy determined in Step 1. Hence, we have for $\underline{y}_{31}=x=\underline{x}_{32}$ :

$$
J_{3}^{(2)}\left(x, \underline{y}_{31}, \underline{x}_{32}, \underline{y}_{32}\right)=-g_{31}+v_{1}(x)=-g_{32}+v_{2}(x) .
$$

- If $x \leq \underline{y}_{31}$, then the optimal strategy would be to switch immediately to regime 1 , and then follow the remaining optimal strategy computed in Step I. Hence, we have for $x \leq \underline{y}_{31}$ :

$$
J_{3}^{(2)}\left(x, \underline{y}_{31}, \underline{x}_{32}, \underline{y}_{32}\right)=-g_{31}+v_{1}(x)
$$

The maximal expected profit in Option 2 is then given by :

$$
v_{3}^{(2)}(x)=\sup _{\left(\underline{y}_{31}, \underline{x}_{32}, \underline{y}_{32}\right) \in \mathcal{D}_{3}^{(2)}} J_{3}^{(2)}\left(x, \underline{y}_{31}, \underline{x}_{32}, \underline{y}_{32}\right) .
$$


- Now, we compare these two options characterized by the values $v_{3}^{(1)}(x)$ and $v_{3}^{(2)}(x)$. First, notice that when $x \leq \underline{y}_{31}^{*}$, no conclusion could be drawn concerning the existence of the switching region $\mathcal{S}_{32}$ as we would obtain $v_{3}^{(1)}(x)=v_{3}^{(2)}(x)$, and the optimal policy in both Options would be to switch directly to regime 1 . We therefore take some $x>\bar{x}_{12}^{*}$ (computed in Step I), which ensures $x>\underline{y}_{31}^{*}$ (albeit to be determined).

- If $v_{3}^{(1)}(x)>v_{3}^{(2)}(x)$, then the switching region $\mathcal{S}_{32}$ does not exist, and there is only one optimal threshold value $\underline{y}_{31}^{*}$ in regime 3 , which is the solution to (6.10). Moreover, as this optimal threshold parameter does not depend on the current state value $x$, once it has been calculated, the computation of the value function $v_{3}\left(x^{\prime}\right)$ in regime 3 for other state $x^{\prime}>0$, is directly derived from the relation :

$$
v_{3}\left(x^{\prime}\right)=J_{3}^{(1)}\left(x^{\prime}, \underline{y}_{31}^{*}\right) .
$$

- If $v_{3}^{(1)}(x) \leq v_{3}^{(2)}(x)$, then the switching region $\mathcal{S}_{32}$ exists. However, solving (6.16) may not yet yield all the three optimal threshold values $\underline{y}_{31}^{*}, \underline{x}_{32}^{*}, \underline{y}_{32}^{*}$ in regime 3 . Indeed, by solving the optimisation problem (6.16), we obtain four different possible outcomes depending on the position of $x$ :

(a) $\operatorname{Argmax} v_{3}^{(2)}(x)=\left\{\left(\underline{y}_{31}, \underline{x}_{32}, \underline{y}_{32}\right): x \leq \underline{y}_{31} \leq \underline{x}_{32} \leq \underline{y}_{32}\right\}$,

(b) $\operatorname{Argmax} v_{3}^{(2)}(x)=\left\{\left(\underline{y}_{31}^{*}, \underline{x}_{32}^{*}, \underline{y}_{32}\right): \underline{y}_{31}^{*}<x<\underline{x}_{32}^{*} \leq \underline{y}_{32}\right\}$,

(c) $\operatorname{Argmax} v_{3}^{(2)}(x)=\left\{\left(\underline{y}_{31}, \underline{x}_{32}, \underline{y}_{32}\right): \underline{y}_{31} \leq \underline{x}_{32} \leq x \leq \underline{y}_{32}\right\}$,

(d) $\operatorname{Argmax} v_{3}^{(2)}(x)=\left\{\left(\underline{y}_{31}, \underline{x}_{32}, \underline{y}_{32}^{*}\right): \underline{y}_{31} \leq \underline{x}_{32} \leq \underline{y}_{32}^{*}<x\right\}$,

As such, solving (6.16) provides us with the relative position of $x$ compared to those threshold values, and in some cases, some optimal threshold values. More precisely, while cases $(a)$ and $(c)$ do not yield the optimal threshold values, in cases $(b)$ and $(d)$, we respectively obtain the threshold values $\left\{\underline{y}_{31}^{*}, \underline{x}_{32}^{*}\right\}$ and $\left\{\underline{y}_{32}^{*}\right\}$. Therefore, we have to solve (6.16) for two different values $x_{b}$ and $x_{d}$ such that $\underline{y}_{31}^{*}<x_{b}<\underline{x}_{32}^{*}$ and $\underline{y}_{32}^{*}<x_{d}$. The difficult point here is that we do not know a priori those optimal threshold values. As such, to obtain two values $x_{b}$ and $x_{d}$ respectively in $\left(\underline{y}_{31}^{*}, \underline{x}_{32}^{*}\right)$ and $\left(\underline{y}_{32}^{*}, \infty\right)$, we shall complete the following iteration. Starting from our initial value $x$, we solve (6.16) and discuss the above four different outcomes respectively:

(a) : actually, since our chosen initial value satisfies $x>\bar{x}_{12}^{*}$, this case could not happen.

(b) : an obvious candidate for $x_{b}$ is $x$ itself. Then, we want to find a candidate $x_{d}$ such that $x_{d}>\underline{y}_{32}^{*}$. Starting with $x_{0}=x$, and $x_{j+1}=2 x_{j}$, we solve successively (6.16) for $x_{j}$ until we get case $(d)$ as the outcome, in other words, until we get a $\bar{j}$ such that $x_{\bar{j}}>\underline{y}_{32}^{*}$. We take $x_{d}=x_{\bar{j}}$.

(d) : an obvious candidate for $x_{d}$ is $x$ itself. Then, we want to find a candidate $x_{b}$ such that $\underline{y}_{31}^{*}<x_{b}<\underline{x}_{32}^{*}$. Setting $x_{0}=x$, and $x_{1}=\frac{x_{0}}{2}$, we solve successively (6.16) for $x_{j}$, starting with $j=1$. If for $x_{j}$, we fall into 
$\star$ case $(c)$ or case $(d)$, then we set $x_{j+1}=\frac{x_{j}}{2}$,

$\star$ case $(b)$, we stop the iteration,

$\star$ case $(a)$, then we set $x_{j+1}=\frac{x_{j}+x_{j-1}}{2}$.

Once our iteration stops, we obtain a $\bar{j}$ such that $\underline{y}_{31}^{*}<x_{\bar{j}}<\underline{x}_{32}^{*}$. We take $x_{b}=x_{j}$.

(c) starting from our initial value, we apply the iterations used in the two previous cases to obtain two satisfying values $x_{b}$ and $x_{d}$.

The resolutions of (6.16) associated with $x_{b}$ and $x_{d}$ give us all the three optimal threshold values.

Moreover, once we have obtained the three optimal threshold values, the computation of the value function $v_{3}\left(x^{\prime}\right)$ in regime 3 for other state values $x^{\prime}>0$, is directly derived from the relation :

$$
v_{3}\left(x^{\prime}\right)=J_{3}^{(2)}\left(x^{\prime}, \underline{y}_{31}^{*}, \underline{x}_{32}^{*}, \underline{y}_{32}^{*}\right)
$$

Summary. To summarize, we have the following algorithm in computing the (at most) five parameters for case B) in Theorem 5.4.

- Fix $x>0$, and compute the function $\left(\bar{x}_{12}, \underline{y}_{21}\right) \rightarrow J_{1}\left(x, \bar{x}_{12}, \underline{y}_{21}\right)$ by (6.4)-(6.5)-(6.6). Then, solve (6.7), which gives the optimal threshold values $\bar{x}_{12}^{*}, \underline{y}_{21}^{*}$ in regimes 1 and 2 .

- Fix $x>\bar{x}_{12}^{*}>0$.

(i) Compute the function $\underline{y}_{31} \rightarrow J_{3}^{(1)}\left(x, \underline{y}_{31}\right)$ by (6.8)-(6.9), and solve $v_{3}^{(1)}(x)$ in (6.10).

(ii) Compute the function $\left(\underline{y}_{31}, \underline{x}_{32}, \underline{y}_{32}\right) \rightarrow J_{3}^{(2)}\left(x, \underline{y}_{31}, \underline{x}_{32}, \underline{y}_{32}\right)$ by (6.11)-(6.12)-(6.13)-(6.14)(6.15), and solve $v_{3}^{(2)}(x)$ in (6.16).

(iii) If $v_{3}^{(1)}(x)>v_{3}^{(2)}(x)$, then the switching region $\mathcal{S}_{32}$ is empty, and the optimal threshold value $\underline{y}_{31}^{*}$ in regime 3 is the solution to (6.10). Otherwise, $\mathcal{S}_{32}$ is nonempty, and the optimal threshold values in regime $3, \underline{y}_{31}^{*}, \underline{x}_{32}^{*}, \underline{y}_{32}^{*}$ are the solutions to (6.16) (solved for two wellchosen values $x_{b}$ and then $x_{d}$, see the above discussion).

Although we have demonstrated here only one case in the three-regime model, the essence remains the same for other cases or even general multi-regime model. What is important here is that the qualitative structure obtained for a general model makes it possible to turn the problem into a few finite-dimensional mathematical programming problems, which are very easy to solve given the vast choices of optimization software packages.

\section{Appendix : Computation of functionals of expected hitting times for GBM}

In this appendix we demonstrate, via the GBM, how to compute the expectation functionals involved in Section 6. Consider the GBM

$$
X_{t}^{x}=x e^{\mu t+W_{t}}, \quad t \geq 0, x>0
$$


with $\mu \in \mathbb{R}$. We compute explicitly the expectation functionals defined in (6.2) and (6.3).

\section{Lemma A.1}

$$
\begin{aligned}
R(x, a) & =\left\{\begin{array}{l}
\left(\frac{a}{x}\right)^{\mu-\sqrt{\mu^{2}+2 \rho}}, \quad \text { if } a \geq x \\
\left(\frac{a}{x}\right)^{\mu+\sqrt{\mu^{2}+2 \rho}}, \quad \text { if } 0<a<x,
\end{array}\right. \\
\hat{R}(x, a, b) & =R(x, a) R(a, b), \\
\text { and } \quad \tilde{R}(x, a, b) & =\left(\frac{a}{x}\right)^{\mu} \frac{\operatorname{sh}\left[\log \frac{b}{x} \sqrt{2 \alpha+\mu^{2}}\right]}{\operatorname{sh}\left[\log \frac{b}{a} \sqrt{2 \alpha+\mu^{2}}\right]}, \text { if } a \leq x \leq b .
\end{aligned}
$$

Proof. The first relation is evident from the fact that for the GBM (A.1), the hitting time $\tau_{a}^{x}$ is written as $\tau_{a}^{x}=\inf \left\{t \geq 0: \mu t+W_{t}=\ln \left(\frac{a}{x}\right)\right\}$, and so

$$
E\left[e^{-\rho \tau_{a}^{x}}\right]=e^{\mu \log \left(\frac{a}{x}\right)-\left|\log \frac{a}{x}\right| \sqrt{\mu^{2}+2 \rho}} .
$$

(see, e.g. p. 223, 2.0.1 in [3].) For the second one, we use the Lévy property of the Brownian motion, and write

$$
\begin{aligned}
E\left[e^{-\rho \tau_{a b}^{x}}\right] & =\int_{0}^{\infty} E\left[e^{-\rho \tau_{a b}^{x}} \mid \tau_{a}^{x}=s\right] p_{\tau_{a}^{x}}(s) d s \\
& =\int_{0}^{\infty} E\left[e^{-\rho\left(s+\tau_{b}^{a}\right)}\right] p_{\tau_{a}^{x}}(s) d s,
\end{aligned}
$$

where $p_{\tau_{a}^{x}}$ is the probability density function of $\tau_{a}^{x}$. Therefore,

$$
\begin{aligned}
E\left[e^{-\rho \tau_{a b}^{x}}\right] & =E\left[e^{-\rho \tau_{b}^{a}}\right] \int_{0}^{\infty} e^{-\rho s} p_{\tau_{a}^{x}}(s) d s \\
& =E\left[e^{-\rho \tau_{b}^{a}}\right] E\left[e^{-\rho \tau_{a}^{x}}\right] .
\end{aligned}
$$

Finally, for the third relation, we refer to p. 233, 3.0.5, in [3].

\section{Lemma A.2}

$$
\begin{aligned}
& F(f ; x, a)= \begin{cases}\frac{1}{\sqrt{2 \pi t}} \int_{0}^{\infty} e^{-\rho t} \int_{0}^{a} \frac{f(y)}{y} e^{\frac{-\left(\log \frac{y}{x}-\mu t\right)^{2}}{2 t}}\left[1-e^{\frac{-2 \log \frac{a}{x} \cdot \log \frac{a}{y}}{t}}\right] d y d t, & \text { if } x \leq a \\
\frac{1}{\sqrt{2 \pi t}} \int_{0}^{\infty} e^{-\rho t} \int_{a}^{\infty} \frac{f(y)}{y} e^{\frac{-\left(\log \frac{y}{x}-\mu t\right)^{2}}{2 t}}\left[1-e^{\frac{-2 \log \frac{a}{x} \cdot \log \frac{a}{y}}{t}}\right] d y d t, & \text { if } 0<a<x\end{cases} \\
& \hat{F}(f ; x, a, b)=R(x, a) F(f ; x, b), \\
& \text { and } \tilde{F}(f ; x, a, b)=\frac{1}{\sqrt{2 \pi t}} \int_{0}^{\infty} e^{-\rho t} \int_{a}^{b} \frac{f(y)}{y} e^{\mu \log \frac{y}{x}-\frac{\mu^{2} t}{2}} \\
& \sum_{k=-\infty}^{\infty}\left(e^{-\left(\log \frac{y}{x}+2 k \log \frac{b}{a}\right)^{2} / 2 t}-e^{-\left(\log \frac{y}{a}+2 k \log \frac{b}{a}\right)^{2} / 2 t}\right) d y, \quad \text { if } a \leq x \leq b .
\end{aligned}
$$


Proof. 1) (i) Consider first the case where $x \leq a$, and denote $W_{t}^{\mu}=\mu t+W_{t}, \bar{M}_{t}=$ $\max _{0 \leq s \leq t} W_{s}^{\mu}$. Then for any $t \geq 0$ and $0 \leq y \leq a$ :

$$
\begin{aligned}
P\left(\tau_{a}^{x} \geq t, X_{t}^{x} \geq y\right)= & P\left(\max _{0 \leq s \leq t} X_{s}^{x} \leq a, X_{t}^{x} \geq y\right) \\
= & P\left(\bar{M}_{t} \leq \log \frac{a}{x}, W_{t}^{\mu} \geq \log \frac{y}{x}\right) \\
= & P\left(W_{t}^{\mu} \geq \log \frac{y}{x}\right)-P\left(\bar{M}_{t}>\log \frac{a}{x}, W_{t}^{\mu} \geq \log \frac{y}{x}\right) \\
= & \frac{1}{\sqrt{2 \pi t}} \int_{\log \frac{y}{x}}^{\infty} e^{-\frac{(z-\mu t)^{2}}{2 t}} d z-\frac{1}{\sqrt{2 \pi t}} \int_{\log \frac{y}{x}}^{\infty} e^{\mu z-\frac{\mu^{2} t}{2}-\frac{\left(\left|z-\log \frac{a}{x}\right|+\log \frac{a}{x}\right)^{2}}{2 t}} d z \\
& (\operatorname{see} p \cdot 198,1.1 .8 \operatorname{in}[3]) \\
= & \frac{1}{\sqrt{2 \pi t}} \int_{\log \frac{y}{x}}^{\log \frac{a}{x}} e^{-\frac{(z-\mu t)^{2}}{2 t}}\left[1-e^{\left.-\frac{2 \log \frac{a}{x}\left(\log \frac{a}{x}-z\right)}{t}\right]}\right] d z .
\end{aligned}
$$

Next, let $p_{t}(s, y)$ be the joint density function of $\left(\tau_{a}^{x}, X_{t}^{x}\right)$. Then,

$$
\begin{aligned}
E \int_{0}^{\tau_{a}^{x}} e^{-\rho t} f\left(X_{t}^{x}\right) d t & =\int_{0}^{a} \int_{0}^{\infty} \int_{0}^{s} e^{-\rho t} f(y) p_{t}(s, y) d t d s d y \\
& =\int_{0}^{a} f(y) \int_{0}^{\infty} e^{-\rho t} \int_{t}^{\infty} p_{t}(s, y) d t d s d y \\
& =\int_{0}^{a} f(y) \int_{0}^{\infty} e^{-\rho t} P\left(\tau_{a}^{x} \geq t, X_{t}^{x} \in d y\right) d t
\end{aligned}
$$

However, by the earlier calculation

$$
\begin{aligned}
P\left(\tau_{a}^{x} \geq t, X_{t} \in d y\right) & =-d P\left(\tau_{a}^{x} \geq t, X_{t}^{x} \geq y\right) \\
& =\frac{1}{\sqrt{2 \pi t} y} e^{\frac{-\left(\log \frac{y}{x}-\mu t\right)^{2}}{2 t}}\left[1-e^{\frac{-2 \log \frac{a}{x} \cdot \log \frac{a}{y}}{t}}\right] d y,
\end{aligned}
$$

which proves the required result.

(ii) Suppose that $0<a<x$, and let $\underline{M}_{t}=\inf _{0 \leq s \leq t} W_{s}^{\mu}$. Then, for any $t \geq 0$ and $y \geq a$ :

$$
\begin{aligned}
P\left(\tau_{a}^{x} \geq t, X_{t}^{x} \leq y\right)= & P\left(\underline{M}_{t} \geq \log \frac{a}{x}, W_{t}^{\mu} \leq \log \frac{y}{x}\right) \\
= & P\left(W_{t}^{\mu} \leq \log \frac{y}{x}\right)-P\left(\underline{M}_{t}<\log \frac{a}{x}, W_{t}^{\mu} \geq \log \frac{y}{x}\right) \\
= & \frac{1}{\sqrt{2 \pi t}} \int_{-\infty}^{\log \frac{y}{x}} e^{-\frac{(z-\mu t)^{2}}{2 t}} d z-\frac{1}{\sqrt{2 \pi t}} \int_{-\infty}^{\log \frac{y}{x}} e^{\mu z-\frac{\mu^{2} t}{2}-\frac{\left(\left|z-\log \frac{a}{x}\right|-\log \frac{a}{x}\right)^{2}}{2 t}} d z \\
& (\operatorname{see} p \cdot 199,1.2 .8 \operatorname{in}[3]) \\
= & \frac{1}{\sqrt{2 \pi t}} \int_{\log \frac{a}{x}}^{\log \frac{y}{x}} e^{-\frac{(z-\mu t)^{2}}{2 t}}\left[1-e^{-\frac{2 \log \frac{a}{x}\left(\log \frac{a}{x}-z\right)}{t}}\right] d z,
\end{aligned}
$$

and we get the required result as in (i).

2) By using the Lévy property of the Brownian motion, we have

$$
E\left[\int_{\tau_{a}^{x}}^{\tau_{a b}^{x}} e^{-\rho t} f\left(X_{t}^{x}\right) d t\right]=\int_{0}^{\infty} E\left(\int_{s}^{s+\tau_{b}^{a}} e^{-\rho t} f\left(X_{t}^{x}\right) d t\right) p_{\tau_{a}^{x}}(s) d s
$$




$$
\begin{aligned}
& =\int_{0}^{\infty} E\left(\int_{0}^{\tau_{b}^{a}} e^{-\rho(t+s)} f\left(X_{t}^{x}\right) d t\right) p_{\tau_{a}^{x}}(s) d s \\
& =\int_{0}^{\infty} e^{-\rho t} F(f ; x, b) p_{\tau_{a}^{x}}(s) d s \\
& =E\left[e^{-\rho \tau_{a}^{x}}\right] F(f ; x, b)=R(x, a) F(f ; x, b) .
\end{aligned}
$$

3) We now consider the third relation, and denote $\underline{M}_{t}=\min _{0 \leq s \leq t} W_{s}^{\mu}$. Then for any $t \geq 0$, $a \leq y \leq b$, and $a \leq x \leq b$ :

$$
\begin{aligned}
P\left(\tau_{a}^{x} \wedge \tau_{b}^{x} \geq t, X_{t}^{x} \geq y\right) & =P\left(\max _{0 \leq s \leq t} X_{s}^{x} \leq b, \min _{0 \leq s \leq t} X_{s}^{x} \geq a, X_{t}^{x} \geq y\right) \\
& =P\left(\bar{M}_{t} \leq \log \frac{b}{x}, \underline{M}_{t} \geq \log \frac{a}{x}, W_{t}^{\mu} \geq \log \frac{y}{x}\right) \\
& =\frac{1}{\sqrt{2 \pi t}} \int_{\log \frac{y}{x}}^{\infty} e^{\mu z-\frac{\mu^{2} t}{2}} \\
& \sum_{k=-\infty}^{\infty}\left(e^{-\left(z+2 k \log \frac{b}{a}\right)^{2} / 2 t}-e^{-\left(z-\log \frac{a}{x}+2 k \log \frac{b}{a}\right)^{2} / 2 t}\right) d z ;
\end{aligned}
$$

see p. $212,1.15 .8$ in [3]. Next, let $q_{t}(s, y)$ be the joint density function of $\left(\tau_{a}^{x} \wedge \tau_{b}^{x}, X_{t}^{x}\right)$. Then, we have

$$
\begin{aligned}
E \int_{0}^{\tau_{a}^{x} \wedge \tau_{b}^{x}} e^{-\rho t} f\left(X_{t}^{x}\right) d t & =\int_{a}^{b} \int_{0}^{\infty} \int_{0}^{s} e^{-\rho t} f(y) q_{t}(s, y) d t d s d y \\
& =\int_{a}^{b} f(y) \int_{0}^{\infty} e^{-\rho t} \int_{t}^{\infty} q_{t}(s, y) d s d t d y \\
& =\int_{a}^{b} f(y) \int_{0}^{\infty} e^{-\rho t} P\left(\tau_{a}^{x} \wedge \tau_{b}^{x} \geq t, X_{t}^{x} \in d y\right) d t
\end{aligned}
$$

Now, by the earlier calculation

$$
\begin{aligned}
P\left(\tau_{a}^{x} \wedge \tau_{b}^{x} \geq t, X_{t} \in d y\right)= & -d P\left(\tau_{a}^{x} \wedge \tau_{b}^{x} \geq t, X_{t}^{x} \geq y\right) \\
= & \frac{1}{\sqrt{2 \pi t} y} e^{\mu \log \frac{y}{x}-\frac{\mu^{2} t}{2}} \\
& \sum_{k=-\infty}^{\infty}\left(e^{-\left(\log \frac{y}{x}+2 k \log \frac{b}{a}\right)^{2} / 2 t}-e^{-\left(\log \frac{y}{a}+2 k \log \frac{b}{a}\right)^{2} / 2 t}\right),
\end{aligned}
$$

from which we get the required result.

\section{References}

[1] Bayraktar E. and M. Egami (2007) : "On the optimal switching problem for one dimensional-diffusions", preprint, University of Michigan. 
[2] Bensoussan A. and J.L. Lions (1982) : Contrôle impulsionnel et inéquations variationnelles, Dunod.

[3] Borodin A. and P. Salminen (1996) : Handbook of Brownian Motion, Facts and Formulae, Birkhauser.

[4] Brekke K. and B. Oksendal (1994) : "Optimal switching in an economic activity under uncertainty", SIAM J. Cont. Optim., 32, 1021-1036.

[5] Brennan M. and E. Schwartz (1985) : "Evaluating natural resource extraction", J. Business, 58, 135-137.

[6] Carmona R. and M. Ludkowski (2005) : "Optimal switching with applications to energy tolling agreements", preprint, Princeton university.

[7] Dayanik S. and I. Karatzas (2003) : "On the optimal stopping problem for onedimensional diffusions", Stochastic Process. Appl., 107, 173-212.

[8] Deng S. and Z. Xia (2005) : "Pricing and hedging electric supply contracts : a case with tolling agreements", preprint.

[9] Dixit A. (1989) : "Entry and exit decisions under uncertainty", J. Political Economy, 97, 620-638.

[10] Djehiche B., Hamadène S. and A. Popier (2007) : "A finite horizon optimal switching problem", preprint.

[11] Duckworth K. and M. Zervos (2001) : "A model for investment decisions with switching costs", Annals of Applied Probability, 11, 239-250.

[12] El Karoui N. (1981) : Les aspects probabilistes du contrôle stochastique, Lect. Notes in Math., Springer Verlag.

[13] Guo X. and P. Tomecek (2007) : "Connections between singular control and optimal switching", to appear in SIAM J. Cont. Optim.

[14] Hamadène S. and M. Jeanblanc (2007) : "On the starting and stopping problem : application in reversible investment", Math. Oper. Res., 32, 182-192.

[15] Hu Y. and S. Tang (2007) : "Multi-dimensional BSDE with oblique reflection and optimal switching", preprint.

[16] Pham H. (2007) : "On the smooth-fit property for one-dimensional optimal switching problem", Séminaire de Probabilités, Vol. XL, 187-201.

[17] Pham H. and V. Ly Vath (2007) : "Explicit solution to an optimal switching problem in the two-regime case", SIAM J. Cont. Optim., 46, 395-426.

[18] Tang S. and J. Yong (1993) : "Finite horizon stochastic optimal switching and impulse controls with a viscosity solution approach", Stoch. and Stoch. Reports, 45, 145-176. 
[19] Zervos M. (2003) : "A problem of sequential entry and exit decisions combined with discretionary stopping", SIAM J. Cont. Optim., 42, 397-421. 